\title{
Spatial wavenumber selection in recurrent precipitation
}

\author{
Ryan Goh \\ Department of Mathematics \\ Michigan State University \\ East Lansing, MI 48824
}

\author{
Samantha Mesuro \\ Harvey Mudd College \\ 301 Platt Boulevard \\ Claremont, CA 91711
}

\author{
Arnd Scheel \\ University of Minnesota \\ School of Mathematics \\ 206 Church St. S.E. \\ Minneapolis, MN 55455
}

\begin{abstract}
We study invasion fronts in a class of simple, two-species reaction-diffusion systems that occur as models for recurrent precipitation and undercooled liquids. We exhibit several different modes of front propagation: the invasion of an unstable homogeneous equilibrium can create persistent periodic patterns, transient patterns, or simply a homogeneous state. We give criteria that distinguish between these different modes of invasion, corroborate our predictions with numerical simulations, and point to a rich variety of more subtle phenomena and bifurcations.
\end{abstract}

Running head: Wavenumber selection in precipitation

Corresponding author: Arnd Scheel

Keywords: front propagation, pattern formation, phase-field system, Liesegang patterns, pulled and pushed fronts

MSC 2010 Classification: 35B36, 37L15 


\section{Introduction}

We study patterns that nucleate from a spatially homogeneous, unstable state in a large system, when perturbations to the unstable state are spatially localized. The patterns that emerge in this situation are typically created by fronts that invade the unstable state. Our focus is on a seemingly simple, yet surprisingly rich example, that extends the well-understood scalar Nagumo or Allen-Cahn equation by an additional neutral field.

Consider, for instance, the simple reaction-diffusion model of a closed system, $C \rightleftharpoons E$, with reaction rate $f(c, e)$,

$$
\begin{aligned}
& c_{t}=c_{x x}-f(c, e) \\
& e_{t}=\kappa e_{x x}+f(c, e) .
\end{aligned}
$$

Here, we think of an idealized unbounded reactor $x \in \mathbb{R}$, diffusivity $\kappa<1$ for the species $e$, and diffusivity of species $c$ scaled to 1 . We are particularly interested in the case when the nullclines of $f$ possess threshold characteristic, such as in the prototypical example

$$
f(c, e)=g(e)+\gamma c, \quad g(e)=e(1-e)(e-1 / 2), \quad \gamma \in \mathbb{R} .
$$

The substitution $\theta=c+\kappa e$ readily yields a conservation law for the total mass $c+e$, with flux $\theta$, which is known in other contexts as the phase-field system,

$$
\begin{aligned}
\theta_{t}+l e_{t} & =\theta_{x x} \\
e_{t} & =\kappa e_{x x}+g(e)+\gamma(\theta-\kappa e) .
\end{aligned}
$$

In that context, $\theta$ is a temperature field, $e$ an order parameter, and $l=1-\kappa$ the (positive) latent heat; see for instance [13] for some background.

In the form (1.1), the system has also been used as a model for recurrent precipitation, interpolating between super-saturation models as in [21] and phenomenological models such as the Cahn-Hilliard equation [7]. One seeks, for instance, to explain formation of Liesegang bands. The bands form when a moving reaction front creates a reaction product that precipitates, forming distinct bands; see [7, 17, 19, 21] for background, motivation and phenomena.

We will work with the system (1.1) with $\kappa<1$ and $f$ the simple cubic,

$$
\begin{aligned}
& c_{t}=c_{x x}-e(1-e)(e-a)-\gamma c \\
& e_{t}=\kappa e_{x x}+e(1-e)(e-a)+\gamma c .
\end{aligned}
$$

Many of our results remain true at least qualitatively for more general forms of nonlinearities $f$. The parameter $a$ is convenient for a parameterization of equilibria, but $a$ can be formally eliminated by shifting $c$ and $e$. Throughout, we mostly focus on the case $\gamma>0$ with some attention to the limits $\gamma=0$ and $\gamma=\infty$ : for $\gamma=0$, the $e$-equation decouples and the dynamics are determined by the scalar Allen-Cahn equation; for $\gamma \rightarrow \infty$, one recovers the Cahn-Hilliard equation in an appropriate scaling $[5,8]$.

The specific form $f(c, e)=g(e)+\gamma c, \kappa<1, \gamma>0$, allows a (formal) free energy

$$
W(c, e)=\int_{\mathbb{R}} \frac{\kappa}{2} e_{x}^{2}+G(e)+\frac{\gamma}{2}\left(c^{2}+\frac{\kappa}{1-\kappa}(c+e)^{2}\right) \mathrm{d} x
$$


with $G^{\prime}=-g$. Along smooth solutions, for Neumann or periodic boundary conditions, one verifies that $\frac{\mathrm{d}}{\mathrm{d} t} W(c(t), e(t)) \leq 0$. The system is in fact a gradient flow to this free energy, with metric factor $\mathcal{M}=\mathcal{M}^{*} \geq 0$, so that

$$
\left(\begin{array}{c}
c_{t} \\
e_{t}
\end{array}\right)=-\mathcal{M} \nabla_{L^{2}} W, \quad \mathcal{M}=\left(\begin{array}{cc}
\mathrm{id}-\frac{1-\kappa}{\gamma} \partial_{x x} & -\mathrm{id} \\
-\mathrm{id} & \mathrm{id}
\end{array}\right) .
$$

With Neumann or periodic boundary conditions, or on the real line, the system (1.3) possesses a curve of spatially homogeneous equilibria $c_{*}=-e_{*}\left(1-e_{*}\right)\left(e_{*}-a\right) / \gamma$. To prepare the statement of our main results, we examine stability of those spatially homogeneous states via the linearized approximation,

$$
\begin{aligned}
& c_{t}=c_{x x}-g^{\prime}\left(e_{*}\right) e-\gamma c \\
& e_{t}=\kappa e_{x x}+g^{\prime}\left(e_{*}\right) e+\gamma c .
\end{aligned}
$$

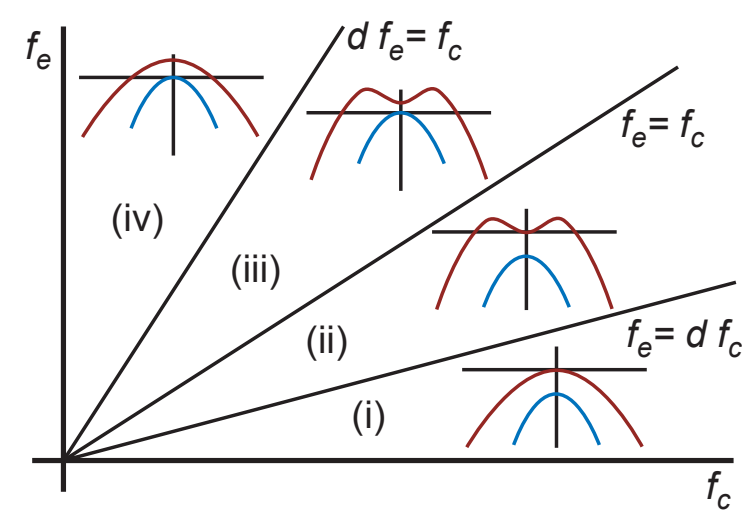

Figure 1.1: The figure shows stability and instability regions of spatially homogeneous equilibria of (1.3) depending on $f_{e}$ and $f_{c}$. The insets show plots of $\lambda_{ \pm}(k)$. Detailed stability information is given in Table 1.

Linear stability of equilibria can be determined explicitly after Fourier transformation,

$$
\left(\begin{array}{c}
c_{t} \\
e_{t}
\end{array}\right)=\left(\begin{array}{cc}
-k^{2}-\gamma & -g^{\prime} \\
\gamma & g^{\prime}-\kappa k^{2}
\end{array}\right)\left(\begin{array}{l}
c \\
e
\end{array}\right),
$$

with two eigenvalues $\lambda_{ \pm}(k)$. When $x \in \mathbb{R}$, all wavenumbers $k \in \mathbb{R}$ are allowed. Depending on $f_{c}=\gamma>0$ and $f_{e}=g^{\prime}$, one can readily determine stability properties of the family of matrices in (1.7). Figure 1.1 shows four qualitatively different regions of stability, depending on the quotient $f_{c} / f_{e}$, which of course is equivalent to the slope of the nullcline of $f(c, e)$. Plotted are those four regions in $f_{c}, f_{e}>0$ and the corresponding eigenvalue curves $\lambda_{ \pm}(k)$,

$$
\lambda_{ \pm}(k)=\frac{1}{2}\left(\operatorname{tr} \pm \sqrt{\operatorname{tr}^{2}-4 \operatorname{det}}\right), \operatorname{tr}=f_{e}-f_{c}-(1+\kappa) k^{2}, \quad \operatorname{det}=\left(\kappa f_{c}-f_{e}\right) k^{2}+\kappa k^{4}, k \in \mathbb{R}
$$

Figure 1.3 shows the phase portraits for $x$-independent solutions of (1.3) with equilibria at $c=-e(1-e)(e-a) / \gamma$. In addition, PDE-stability information is shown, depending on $e$ 

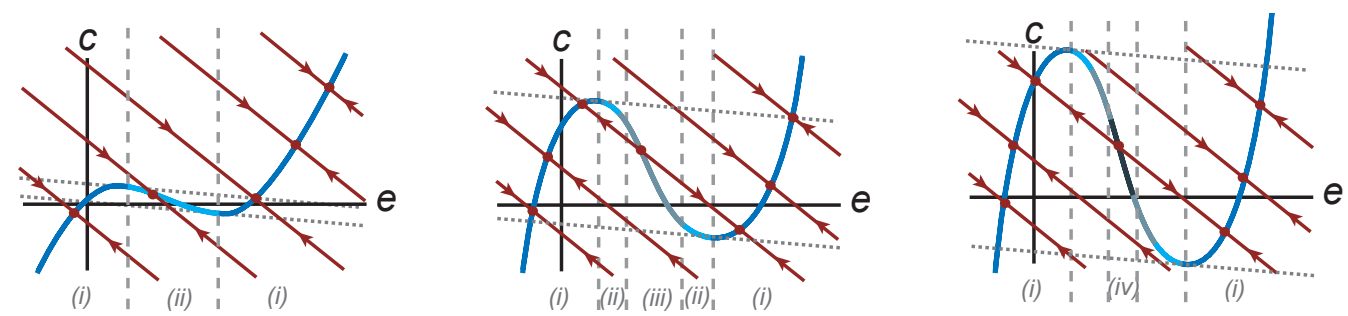

Figure 1.2: Phase portraits are shown for spatially homogeneous dynamics of (1.3) in case $\gamma>1 / 4$, left, and $\kappa / 4<\gamma<1 / 4$, middle, and $\gamma<\kappa / 4$, right. Equilibria are located on the cubic. The dotted vertical lines separate regions of different PDE stability as described in Figure 1.1. Only the righthand picture shows equilibria which are homogeneous unstable of type II. Transitions from stable (i) to spinodal unstable (ii) occur at tangencies with the slanted lines $f_{e}=\kappa f_{c}$.

\begin{tabular}{lcccc}
\hline & ODE-stability & region & $k_{\text {temp }}$ & $k_{\max }$ \\
\hline (i) stable & stable & $f_{c}>\kappa f_{c}>f_{e}$ & 0 & 0 \\
\hline (ii) spinodal & stable & $f_{c}>f_{e}>\kappa f_{c}$ & $k_{\text {temp }}>0$ & $k_{\max }>0$ \\
\hline (iii) homogeneous unstable I & unstable & $f_{e}>f_{c}>\kappa f_{c}$ & $k_{\text {temp }}>0$ & $k_{\max }>0$ \\
\hline (iv) homogeneous unstable II & unstable & $f_{e}>\kappa f_{e}>f_{c}$ & 0 & $k_{\max }>0$ \\
\hline
\end{tabular}

Table 1.1: Stability regions in terms of the gradient $\left(f_{c}, f_{e}\right)$ of the nonlinearity. The wavenumbers $k_{\text {temp }}$ are the wavenumbers with maximal temporal growth. The wavenumbers $k_{\max }$ are the maximal wavenumbers with temporal growth.

and $\gamma$. . The four different stability regions are also described in Table 1.1. There, $k_{\text {temp }}$ is the wavenumber with maximal temporal growth $\lambda_{+}\left(k_{\text {temp }}\right) \geq \lambda_{ \pm}(k)$ for all $k$, and $k_{\max }$ is the maximal unstable wavenumber, $\lambda_{ \pm}(k) \leq 0$ for $|k|>k_{\text {max }}$. One easily calculates the following explicit expressions,

$$
k_{\text {temp }}=\sqrt{\frac{-\kappa\left(f_{c}+f_{e}\right)+\sqrt{\kappa(1+\kappa)^{2} f_{c} f_{e}}}{\kappa(1-\kappa)}}, \quad k_{\max }=\sqrt{\frac{f_{c}-\kappa f_{e}}{\kappa}} .
$$

Finally, ODE stability, which holds in regions (i) and (ii) refers to stability at $k=0$, within the class of $x$-independent solutions.

States with $f_{e}<0$ are stable. In the case $\gamma<0$, complex eigenvalues can occur. The stability in large bounded domains with $L$-periodic (or Neumann) boundary conditions is similar, with dispersion curves being approximated by points where $k \in k_{0} \mathbb{Z}, k_{0}=2 \pi / L$. In particular, the equilibrium $e_{*}=a, c_{*}=0$ is stable when $g^{\prime}(a)=a(1-a)<\kappa \gamma$, and unstable when $a(1-a)>\kappa \gamma$. Varying $a$, we see that homogeneous unstable equilibria exist only when $\gamma>1 / 4$, in the region $|a-1 / 2|<\gamma-1 / 4$.

We are interested in pattern formation from unstable states, that is, from states in regions (ii)-(iv). One can easily check that, possibly shifting $c$ and $e$ and scaling $c, x, t$, we may assume that this unstable state is given by $(c, e)=(0, a)$, with $0<a<1$. Typically, localized 
perturbations evolve into a pair of fronts that propagate to $+\infty$ and $-\infty$, respectively, invading the unstable state and leaving a new state behind.

Our main results characterize three different modes of invasion. These modes are most prominently characterized by the pattern that is being created in the wake of the front. Depending on the parameters $a$ and $\gamma$, fronts can leave behind

(i) a bulk state, spatially constant;

(ii) a periodic pattern, invaded by a bulk state with the same speed;

(iii) a periodic pattern invaded by a bulk state at a slower speed.

The three possibilities are illustrated in space-time plots in Figure 1.3.
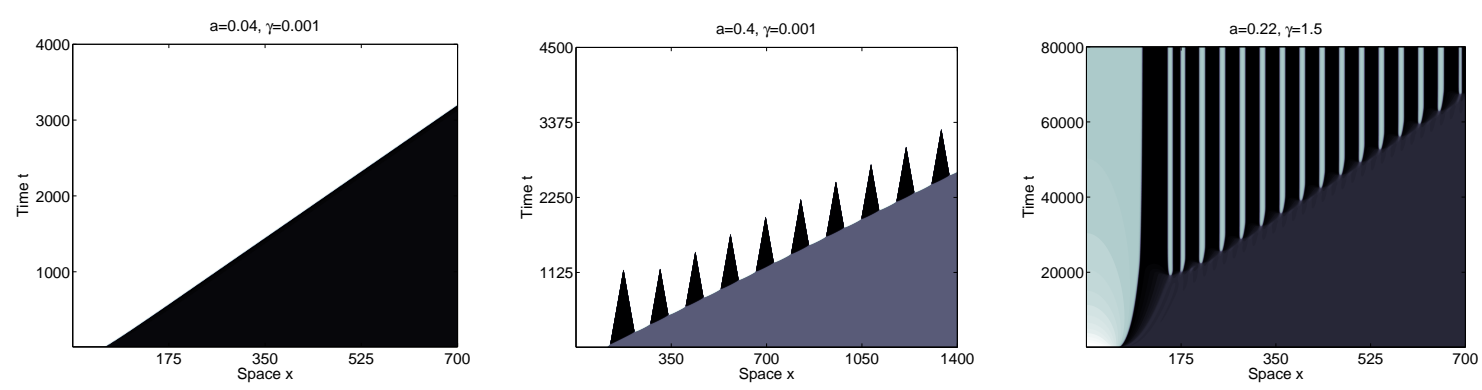

Figure 1.3: Space-time plots of the solution to (1.3). From left to right, we see bulk states, transient patterns, and persistent patterns behind fronts. Initial conditions are perturbations near $x=0$ of the unstable state $c=0, e=a$. Plotted is the value of $e$, with brighter colors corresponding to larger $e$. The parameters are $\gamma=0.001, a=0.04,0.4$ in the left and middle picture, $\gamma=1.5, a=0.22$ in the right picture.

We will refer to the three different modes of invasion as bulk fronts, transient pattern forming fronts, and persistent pattern forming fronts, depending on whether they create a bulk state (i), a transient periodic patterns (ii), or a persistent periodic patterns (iii). We emphasize already here that the term persistent is to be understood relative to the transient patterns. Persistent periodic patterns are unstable and persist only in an idealized situation. They are typically annihilated by secondary invasion fronts. Depending on system parameters, instabilities and secondary invasion fronts may however be very slow, so that patterns appear to actually persist indefinitely in simulations; see Lemma 4.18 and the discussion in Section 5

We next collect some basic intuitive aspects of the invasion process. First, notice that traveling fronts need to conserve mass. To be precise, define the mass of a pattern as the average

$$
m(c, e)=f(c(x)+e(x)) \mathrm{d} x,
$$

whenever the average exists. The mass of the unstable homogeneous state $(c, e)=(0, a)$ therefore is $m=a$. Any pattern $\left(c_{-}, e_{-}\right)(x)$, that results of the invasion process, spatially periodic or spatially homogeneous, possesses the same average $a=f\left(c_{-}(x)+e_{-}(x)\right) \mathrm{d} x$; see Lemma 4.21. 
Depending on the unstable state $a$ and the parameter $\gamma$, one can calculate a linear spreading speed $s_{\operatorname{lin}}(a ; \gamma)$, the largest of all speeds $s$ in which perturbations of the unstable state do not decay in a compact window of a frame moving with speed $s$. Associated with this linear spreading speed are a selected temporal frequency $\omega_{\text {lin }}$, spatial wavenumber $k_{\text {lin }}=\omega_{\text {lin }} / s_{\text {lin }}$, and spatial asymptotics $\mathrm{O}\left(x \mathrm{e}^{\nu_{\operatorname{lin}} x}\right), x \rightarrow \infty$ with spatial mode $\nu_{\text {lin }}$, Re $\nu_{\text {lin }}<0$; see Section 2.1 for precise definitions.

Roughly speaking, nonlinear fronts that agree with the linear prediction are referred to as pulled fronts. Stable nonlinear fronts that are steeper and faster than the linear prediction are referred to as pushed fronts. We refer to Section 3.1 for a more precise characterization of pushed fronts and to Definition 4.9 for a characterization of pulled fronts. Both characterizations differ slightly from the ones in [35] but coincide in most simple examples.

The first result characterizes the existence of bulk fronts and relates them to the existence of pushed fronts.

Theorem 1 (Bulk Fronts) Let $a_{*}(\kappa)=\min \{\kappa /(2-\kappa), 1 / 3\}$. For any $a<a_{*}(\kappa)$, there is $\gamma_{*}>0$ so that for all $0 \leq \gamma<\gamma_{*}$ there exists a linearly stable front that connects $(c, e)=(0, a)$ with a stable homogeneous equilibrium $(c, e)=(a-1,1)+\mathrm{O}(\gamma)$. This front is a pushed front. For $a>a_{*}(\kappa)$ and $\gamma \geq 0$, sufficiently small, such pushed fronts do not exist.

We refer to Section 3 for precise statements and proofs.

In fact, for $\gamma>0$, small, we have $\omega_{\text {lin }}, k_{\text {lin }}>0$, so that the linear analysis predicts oscillatory front propagation and the formation of patterns in the wake of the front. The above pushed fronts can therefore be viewed as a nonlinear mechanism that eliminates patterns by means of a faster and steeper nonlinear mode of propagation.

Our second result characterizes robustness of modulated fronts. Robustness refers to a property of the linearization at such an invasion front. Roughly speaking, it states that the linearization at such a front is typically onto. Failure of robustness refers to situations where this linearization necessarily possesses a cokernel.

Theorem 2 (Transient versus persistent patterns) Fronts that invade an unstable state and leave behind a persistent periodic pattern are robust if and only if that periodic pattern is stable when considered with periodic boundary conditions of period $2 \pi / k_{\text {lin }}$, corresponding to its minimal period. In particular, we can expect persistent pattern forming fronts only if stable periodic patterns with mass a and selected wavenumber $k_{\text {lin }}$ exist.

We refer to Section 4 for precise statements and proofs.

The possibility of persistent pattern forming fronts is somewhat surprising since the periodic pattern created in the wake of the invasion process is unstable. In fact, in our system (1.3), periodic patterns are always unstable when considered on unbounded domains, or with periodic boundary conditions that are multiples of the period of the pattern [25]. In that respect, the invasion process does not correspond to the intuition where the spatial competition between a stable state and an unstable state leads to invasion and elimination of the unstable state. 
Corollary 4.15 contains a general statement on robustness of invasion fronts. It turns out that invasion fronts can create unstable patterns as long as the instability in the wake is not a harmonic of the frequency of the invasion process.

Remark 1.1 We emphasize that the fronts that we are interested in, here, are quite different from the fronts in phase-field models that have been studied previously; see for instance [1, 6, 14, 15]. Interfaces (or fronts) studied in these works separate (or connect) two stable homogeneous states, while we are interested in fronts where at least one of the asymptotic states is unstable. Pushed and pulled fronts in the phase-field system have been studied in [9], albeit under a somewhat different perspective. Pulled fronts play a significant role in a variant of the system under consideration, here, studied in [16]. There, invasion of a metastable state involves a transient, unstable intermediate state, from which a stable state forms through a secondary front. Different from the study here, only pulled fronts have been observed; see also [36].

Remark 1.2 Transient unstable patterns in the wake of invasion fronts have been observed in [33, 34]. The $\lambda-\omega$ systems studied there describe invasion of an unstable state near a Hopf bifurcation. The patterns selected by the leading edge are periodic but moving, $u=u(k x-\omega t)$, $\omega \neq 0$. Similar to our situation, those selected patterns are unstable for a wide range of parameter values. The instability is quite different from the instability here, often creating spatio-temporally chaotic states. The results in [33] give criteria for the creation and length of the transient in the wake of an invasion front.

Outline: In Section 2, we characterize linear spreading speeds and selected wavenumbers. In Section 3, we consider bulk fronts in the limit $\gamma \sim 0$ of weak coupling. In Section 4 , we study robustness using counting arguments for Fredholm indices. Section 5 contains a discussion of our results, some extensions, and numerical simulations that point to a number of other interesting bifurcations.

Acknowledgments The authors gratefully acknowledge support by the National Science Foundation under grant NSF-DMS-0806614.

\section{Linear spreading speeds}

The goal of this section is to determine linear spreading speeds, selected frequencies, and spatial decay rates. We therefore start by giving some precise background on linear spreading speeds in Section 2.1. We will describe the ideas in the context of a general reaction-diffusion system since there does not appear to be a good reference for the view point taken here. In particular, we derive a system of polynomial equations for the unknowns $s_{\text {lin }}>0, \omega_{\text {lin }} \geq 0$, and $\nu_{\text {lin }} \in \mathbb{C}$. In Section 2.2, we then relate this general criterion for linear spreading speeds and pointwise growth to the notion of absolute spectrum [27] in order to define more general absolute spreading speeds. We then specify to our particular case, Section 2.3, and calculate linear spreading speeds perturbatively in two limiting regimes, Sections 2.4 and 2.5. Finally, in Section 2.6, we present numerical results that give spreading speeds as a function of $a$ and $\gamma$ for selected values of $\kappa$. 


\subsection{Double roots and pinching}

Growth of perturbations of a spatially homogeneous equilibrium is governed by the linearized equation at small amplitudes. When studying the spatial spreading of initially localized perturbations, one investigates the linearized equation in a moving frame of speed $s$. One then determines whether in this fixed frame of reference, smooth and localized initial conditions decay pointwise, that is, in any fixed finite-size window $x \in[-L, L]$. The supremum of all speeds $s$ for which we do not observe pointwise decay is then referred to as the linear spreading speed.

To be precise, consider the reaction diffusion system

$$
u_{t}=D u_{x x}+F(u) \in \mathbb{R}^{N},
$$

with smooth kinetics $F$ and positive diffusion matrix $D+D^{*}>0$. We assume that $u(x) \equiv 0$ is an equilibrium, $F(0)=0$, with linearization in a comoving frame $\xi=x-s t$ given by

$$
u_{t}=D u_{\xi \xi}+s u_{\xi}+F^{\prime}(0) u
$$

The dispersion relation is obtained from the ansatz $u(t, x)=u_{0} \mathrm{e}^{\lambda t+\nu \xi}$ in $(2.2)$,

$$
d_{s}(\lambda, \nu)=d(\lambda-s \nu, \nu), \quad d(\lambda, \nu)=\operatorname{det}\left(D \nu^{2}+F^{\prime}(0)-\lambda\right) .
$$

We assume that 0 is unstable, that is, $d(\lambda, \mathrm{i} k)=0$ for some $\operatorname{Re} \lambda>0, k \in \mathbb{R}$.

Pointwise instability in the comoving frame is induced by double roots (or branch points, depending on which variable one is solving for), rather than simple roots with $\nu \in \mathrm{i} \mathbb{R}$,

$$
d_{s}\left(\lambda_{*}, \nu_{*}\right)=0, \quad \partial_{\nu} d_{s}\left(\lambda_{*}, \nu_{*}\right)=0 .
$$

In fact, using the pointwise representation of the fundamental solution to (1.6), one can see that pointwise growth is induced by double roots of the dispersion relation, which satisfy an additional pinching condition: $\nu_{ \pm}(\lambda) \rightarrow \pm \infty$ as $\lambda \rightarrow+\infty$, where the functions $\nu_{ \pm}(\lambda)$ are continuations of the two roots with $\nu_{ \pm}\left(\lambda_{*}\right)=\nu_{*}[4,3]$. Therefore, when decreasing $s$ past the linear spreading speed, such a branchpoint of the dispersion relation crosses the imaginary axes. Summarizing, we define spreading speeds as follows.

Definition 2.1 (Linear spreading speeds) We define the linear spreading speed as the largest value $s$ for which there is a solution to

$$
d_{s}(\mathrm{i} \omega, \nu)=0, \quad \partial_{\nu} d_{s}(\mathrm{i} \omega, \nu)=0, \quad \nu_{ \pm}(\lambda) \rightarrow \pm \infty \text { for } \lambda \rightarrow \infty .
$$

We will refer to the frequency of the neutral mode $\operatorname{Im} \lambda=: \omega_{\operatorname{lin}}$ as the linear frequency of the invasion process, to $\nu=: \nu_{\operatorname{lin}}$ as the spatial asymptotics, and to $k_{\operatorname{lin}}=: \omega_{\operatorname{lin}} / s_{\operatorname{lin}}$ as the linear selected wavenumber.

Since $\partial_{\nu} d_{s}=-s \partial_{\lambda} d+\partial_{\nu} d$, the second equation can be reformulated as $s=\frac{\mathrm{d} \lambda}{\mathrm{d} \nu}=:-s_{\mathrm{g}}$, with generalized group velocity $s_{\mathrm{g}}$. On the other hand, one often finds that the associated growth 
in the steady frame, $\tilde{\lambda}=\lambda-s \nu$ is real, so that $\lambda-s \nu=0$ and $s=\frac{\lambda}{\nu}=-s_{\mathrm{p}}$, with generalized phase velocity $s_{\mathrm{p}}$. The criterion (2.4) then simplifies to

$$
s_{\mathrm{p}}=s_{\mathrm{gr}},
$$

with a somewhat implicit pinching condition [35]. We will find examples here where the simplified criterion fails since group velocities are not defined, $\partial_{\lambda} d=0$. Also, $\tilde{\lambda}$ will not be real in most cases considered here.

\section{$2.2 \quad$ Absolute spreading speeds}

An alternative notion to the pointwise stability criterion used above is the notion of absolute spectrum, introduced in [27].

Definition 2.2 (Absolute spectrum) [27] The absolute spectrum $\Sigma_{\text {abs }}$ is defined as the set of $\lambda$ such that there exists two roots $\nu$ of $d_{s}(\lambda, \nu)=0$ with equal real part. More precisely, one requires that $\operatorname{Re} \nu_{N}=R e \nu_{N+1}$, where the $2 N$ roots of $d_{s}(\lambda, \nu)$ are repeated with multiplicity and ordered with non-decreasing real part $\operatorname{Re} \nu_{j} \leq \operatorname{Re} \nu_{j+1}$.

Clearly, double roots $\nu_{N}=\nu_{N+1}$ are in the absolute spectrum if the other $2(N-1)$ roots satisfy the above splitting condition. The absolute spectrum captures pointwise instabilities in the following sense. Roughly speaking, the absolute spectrum is the non-discrete part of the limit of spectra of the right-hand side in (2.2) in bounded domains $\xi \in[-L, L]$ when $L \rightarrow \infty$, where of course suitable boundary conditions need to be prescribed at $\xi= \pm L$; see [27] for precise statements and proofs. In that sense, the absolute spectrum captures instabilities in large finite windows with separated boundary conditions, whereas double roots capture instabilities in finite windows as subsets of $x \in \mathbb{R}$.

Definition 2.3 (Absolute spreading speeds) We define the absolute spreading speed as the supremum of speeds $s>0$ for which $\operatorname{Re} \Sigma_{\text {abs }}>0$.

In some cases, one can see that absolute and linear spreading speeds coincide. We refer to Definition 4.12 for a precise characterization of this simple scenario.

Looking at a homotopy $\lambda \rightarrow+\infty$, one concludes that pointwise instability implies instability of the absolute spectrum. Indeed, suppose the double root which causes the pointwise instability does not satisfy the splitting condition. Since for $\lambda \rightarrow+\infty$, there are precisely $N$ roots with $\operatorname{Re} \nu \rightarrow-\infty$ and $N$ roots with $\operatorname{Re} \nu \rightarrow+\infty$, one finds that during the homotopy $\lambda$ necessarily crosses a curve of absolute spectrum, which then necessarily is unstable. In other words, we just showed that

$$
s_{\text {abs }} \geq s_{\text {lin }}
$$

The absolute spectrum can be located by solving

$$
d_{s}(\lambda, \nu)=0, \quad d_{s}(\lambda, \nu+\mathrm{i} \gamma)=0
$$

with $0 \neq \gamma \in \mathbb{R}$, and by tracking the splitting condition. One can follow roots to this complex system of equations for $(\lambda, \nu)$ in $\gamma$, which yields algebraic curves in the complex plane. One 
can show [26] that all curves of absolute spectrum end in double roots, $\gamma=0$, and continue to $\operatorname{Re} \lambda=-\infty$. In particular, the absolute spectrum is connected. Moreover, due to analyticity of (2.7), one can define multiplicities of the absolute spectrum and show that the absolute spectrum depends continuously on system parameters and speed $s$.

Remark 2.4 One can more generally define linear (or absolute) spreading speeds as the set of speeds $s$ for which one observes pointwise instability (or instability of the absolute spectrum). In general, this set need not be connected. Examples are easily constructed near so-called Turing-Hopf bifurcations [31, Def. 2.3], where a homogeneous state is unstable with respect to linear waves $u(k x \mp \omega t), k, \omega \neq 0$, both with non-vanishing group velocity $\pm s_{\mathrm{g}}$. Instability can be observed in frames with speed close to zero or speed close to $\pm s_{\mathrm{g}}$. In such cases, we expect invasion processes to be more subtle, involving transients.

\subsection{Spreading speeds in precipitation kinetics}

We specify the above considerations to the case of precipitation kinetics and find the dispersion relation

$$
d_{s}(\lambda, \nu)=d(\lambda-s \nu, \nu), \quad d(\lambda, \nu)=\lambda^{2}-\left(f_{e}-f_{c}+(1+\kappa) \nu^{2}\right) \lambda-\left(\kappa f_{c}-f_{e}\right) \nu^{2}+\kappa \nu^{4} .
$$

In our case, $f_{c}=\gamma$ and $f_{e}=a(1-a)$. Since the parabolic scaling

$$
f_{c}=r^{2} \cdot \hat{f}_{c}, \quad f_{e}=r^{2} \cdot \hat{f}_{e}, \quad \nu=r \cdot \hat{\nu}, \quad \omega=r^{2} \cdot \hat{\omega}, \quad s=r \cdot \hat{s},
$$

leaves the equations invariant, we may assume $f_{c}=1, f_{e}=1$, or $f_{c}+f_{e}=1$.

Unfortunately, solving the algebraic equations in (2.4) (let alone verifying the pinching condition) for $\nu, \omega, s \in \mathbb{C} \times \mathbb{R} \times \mathbb{R}$ explicitly appears to be impossible. In the following sections, we therefore consider the limits $f_{e}=1, f_{c}=\mu$, and $f_{c}=1, f_{e}=\kappa f_{c}+\mu$, with $\mu>0, \mu \rightarrow 0$. In the last section, we calculate solutions numerically as a function of $f_{c}$, setting $f_{e}=1-f_{c}$.

\subsection{Spreading speeds at weak coupling}

We set $f_{e}=1$ and $f_{c}=\mu$ by rescaling time and space as in (2.9) so that actual spreading speeds are $s_{\text {lin }}=s \sqrt{f_{e}}$ with $s$ computed below. We find

$$
d_{s}(\lambda, \nu)=\left(\nu^{2}+s \nu-\lambda\right)\left(\kappa \nu^{2}+s \nu+1-\lambda\right)-\mu\left(\kappa \nu^{2}+s \nu-\lambda\right) .
$$

For $\mu=0$, the roots $\nu(\lambda)$ are

$$
\nu_{1}^{ \pm}=\frac{1}{2}\left(-s \pm \sqrt{s^{2}+4 \lambda}\right), \quad \nu_{2}^{ \pm}=\frac{1}{2 \kappa}\left(-s \pm \sqrt{s^{2}+4 \kappa(\lambda-1)}\right),
$$

which satisfy $\nu_{j}^{ \pm}(\lambda) \rightarrow \pm \infty$ for $\lambda \rightarrow+\infty$. Possible branch points that satisfy the pinching condition therefore are the four combinations $\nu_{j}^{+}=\nu_{k}^{-}, j, k \in\{1,2\}$. Elementary algebra shows that only two combinations give branch points with $\lambda=0$ so that $\operatorname{Re} \lambda>0$ for $s<s_{*}$ :

(i) $s_{22}= \pm 2 \sqrt{\kappa}, \nu_{2}^{-}=\nu_{2}^{+}=-s_{22} /(2 \kappa), \lambda=0$; 
(ii) $s_{12}= \pm \frac{1}{\sqrt{1-\kappa}}, \nu_{1}^{-}=\nu_{2}^{+}=-s_{12}, \lambda=0$, when $\kappa<\frac{1}{2}$.

Since $\left|s_{12}\right|>\left|s_{22}\right|$ for $\kappa<\frac{1}{2}$, the actual spreading speed is

$$
s_{\operatorname{lin}}= \begin{cases}\frac{1}{\sqrt{1-\kappa}}, & \text { for } 0<\kappa<\frac{1}{2} ; \\ 2 \sqrt{\kappa}, & \text { for } \frac{1}{2} \leq \kappa \leq 1\end{cases}
$$

We note that the spreading speed of instabilities is nonzero in the limit when $\kappa \rightarrow 0$. Also, $\nu \in \mathbb{R}$ at the double root, that is, the instability generates a uniform pattern with wavenumber $k=0$ in the uncoupled limit $\gamma=0$.

We next study the effect of weak coupling, $0<f_{c}=\mu \ll 1$. In each case $\kappa<1 / 2, \kappa=$ $1 / 2, \kappa>1 / 2$, it is sufficient to follow double roots locally, that is, to use perturbation analysis for double roots in $\mu$.

The case $\kappa>1 / 2$. We locally expand in $\hat{\nu}=\nu+1 / \sqrt{\kappa}, \hat{s}=s-2 \sqrt{\kappa}, \lambda$, and $\mu$,

$d_{s}(\lambda, \nu)=-\frac{2 \kappa-1}{\kappa} \lambda-(2 \kappa-1) \hat{\nu}^{2}+\mu+\frac{2 \kappa-1}{\kappa^{3 / 2}} \hat{s}+\mathrm{O}\left(|\mu|^{2}+|\hat{s}|^{2}+|\lambda|^{2}+|\hat{\nu}|^{2}(|\hat{\mu}|+|\hat{s}|+|\lambda|)+|\hat{\nu}|^{3}\right)$.

Solving $\partial_{\nu} d_{s}=0$ for $\hat{\nu}$ and substituting the result into the equation $d_{s}=0$, one readily finds the expansion for the spreading speed

$$
s_{\operatorname{lin}}(\mu)=2 \sqrt{\kappa}-\frac{\kappa^{3 / 2}}{2 \kappa-1} \mu+\mathrm{O}\left(\mu^{2}\right), \quad \kappa>1 / 2
$$

The case $\kappa<1 / 2$. The case $\kappa<1 / 2$ is slightly more difficult. In fact, $\partial_{\lambda} d_{s}$ vanishes at the double root, so that for $\mu \neq 0$ we have two double roots that collide in the degenerate limit $\mu \rightarrow 0$. In order to derive a local expansion, we set $\hat{\nu}=\nu+1 / \sqrt{1-\kappa}, \hat{s}=s-1 / \sqrt{1-d}$, and find

$$
\begin{aligned}
d_{s}(\lambda, \nu)= & \lambda^{2}-\frac{1-2 \kappa}{1-\kappa} \hat{\nu}^{2}+\frac{2 \kappa}{\sqrt{1-\kappa}} \lambda \hat{\nu}+\mu+\frac{1}{1-\kappa} \hat{s}^{2}+\frac{2 \kappa}{1-\kappa} \hat{\nu} s+\frac{2}{\sqrt{1-\kappa}} \lambda \hat{s} \\
& +\mathrm{O}\left(|\mu|(|\hat{s}|+|\hat{\nu}|+|\lambda|)+\left|\mu^{2}\right|+|\hat{\nu}|^{3}+|\lambda|^{3}+|\hat{s}|^{3}\right) .
\end{aligned}
$$

We can solve the equation $\partial_{\nu} d_{s}(\lambda, \nu)=0$ for $\nu$ with the implicit function theorem and obtain

$$
\hat{\nu}=\nu_{*}(\lambda, \hat{s}, \mu)=\frac{\kappa \sqrt{1-\kappa}}{1-2 \kappa} \lambda+\frac{\kappa}{1-2 \kappa} \hat{s}+\mathrm{O}\left(|\mu|+|\lambda|^{2}+|\hat{s}|^{2}\right) .
$$

Substituting this expression into $d_{s}(\lambda, \mu)=0$, we obtain after some manipulations

$$
\frac{(1-\kappa)^{2}}{1-2 \kappa}\left(\lambda+\frac{1}{\sqrt{1-\kappa}} \hat{s}\right)^{2}+\mu+\mathrm{O}\left(|\mu|^{2}+|\mu|(|\lambda|+|\hat{s}|)+|\lambda|^{2}+|\hat{s}|^{2}\right)=0
$$

Setting $\mu=\varepsilon^{2}$ and scaling $\lambda=\varepsilon \lambda_{1}, \hat{s}=\varepsilon s_{1}$, we find

$$
\frac{(1-\kappa)^{2}}{1-2 \kappa}\left(\lambda_{1}+\frac{1}{\sqrt{1-\kappa}} s_{1}\right)^{2}+1+\mathrm{O}(\varepsilon)=0 .
$$


At the linear spreading speed, $\lambda_{1}=\mathrm{i} \omega_{1}$ is purely imaginary. We split real and imaginary part and find for the imaginary part of $(2.13)$

$$
\frac{(1-\kappa)^{3 / 2}}{1-2 \kappa} \omega_{1} s_{1}+\mathrm{O}(\omega \varepsilon)=0
$$

which gives $s_{1}=\mathrm{O}(\varepsilon)$ and $\hat{s}=\mathrm{O}(\mu)$ with the implicit function theorem after dividing by $\omega_{1}$. Substituting into the equation for the real part, we find

$$
-\frac{(1-\kappa)^{2}}{1-2 \kappa} \omega_{1}^{2}+1+\mathrm{O}(\varepsilon)=0
$$

which in turn yields $\omega_{1}= \pm \frac{\sqrt{1-2 \kappa}}{1-\kappa}+\mathrm{O}(\varepsilon)$.

Summarizing, we find linear spreading speeds $s$, leading edge decay $\nu_{\text {lin }}$, and temporal frequency $\omega$ as

$$
\begin{aligned}
s_{\operatorname{lin}} & =\frac{1}{\sqrt{1-\kappa}}+\mathrm{O}(\mu), \\
\omega & =\frac{\sqrt{1-2 \kappa}}{1-\kappa} \sqrt{\mu}+\mathrm{O}(\mu), \\
\nu & =-\frac{1}{\sqrt{1-\kappa}} \pm \mathrm{i} \sqrt{\mu} \frac{\kappa}{\sqrt{1-\kappa} \sqrt{1-2 \kappa}}+\mathrm{O}(\mu),
\end{aligned}
$$

for $f_{c}=\mu>0$.

We note in passing that in the case of $\mu=f_{c}<0$, one finds $\omega=0$ and

$$
s_{\text {lin }}=\frac{1}{\sqrt{1-\kappa}}+\sqrt{\frac{1-2 \kappa}{1-\kappa}} \sqrt{\mu}+\mathrm{O}(\mu) .
$$

In this case, there are actually two spreading speeds, solutions to $s_{\mathrm{ph}}=s_{\mathrm{gr}}$. However, only the larger one, which corresponds to the first instability, is physically relevant.

One can summarize the calculations here as follows. At $f_{c}=0$, there are two double roots that cross the imaginary axis simultaneously at the origin with nonvanishing speed as $s$ decreases. Perturbing, $f_{c} \neq 0$, the two roots split much like a double eigenvalue into either a pair of complex double roots or a pair of real double roots, depending on the sign of $f_{c}$. The pair of double roots, real or complex, crosses the imaginary axis as $s$ decreases. In the case where the eigenvalues are complex, the imaginary part is of order $\sqrt{f_{c}}$ and the real part is of higher order, so that the crossing happens at the same value of $s$ at leading order. Somewhat counterintuitively, the crossing of complex (oscillatory) double roots occurs in the case where the system possesses a gradient structure, $f_{c}=\gamma>0$, while the crossing of real double roots occurs in the regime $f_{c}=\gamma<0$, where there is no Lyapunov function and Hopf bifurcations are known to occur in bounded domains [25].

The case $\kappa \sim 1 / 2$. When $\kappa$ crosses the critical value $\kappa=1 / 2$, we see a crossover from spreading speeds with simple (in $\lambda$ ) double roots, $\kappa>1 / 2$, to a pair of complex double roots crossing at $\kappa<1 / 2$. Without attempting to give complete expansions, here, we sketch the 
main phenomena, only. The leading order expansion of the dispersion relation near $\kappa=1 / 2$ is

$$
\lambda^{2}-\frac{1}{\sqrt{2}} \hat{\nu}^{3}+\sqrt{2} \lambda \hat{\nu}+\mu+2 \hat{s}^{2}+2 \hat{s} \hat{\nu}+2 \sqrt{2} \hat{s} \lambda-2 \hat{\kappa}(\sqrt{2} \hat{s}+\sqrt{2} \hat{\nu}+\lambda)+\text { h.o.t. }=0,
$$

where $\hat{s}=s-\sqrt{2}, \hat{\kappa}=\kappa-1 / 2$, and $\hat{\nu}=\nu+\sqrt{2}$. One can solve the equation $\partial_{\nu} d_{s}=0$ for $\lambda$, which gives

$$
\lambda=\frac{3}{2} \nu^{2}-\sqrt{2} s+2 \kappa+\text { h.o.t. }
$$

Substituting back into the dispersion relation gives an equation for $\nu$ with three roots, and thus three double roots $\lambda_{j}$. For $f_{c}>0$, two of these three double roots are complex conjugate, one is real. The real part of all double roots is increasing as $s$ decreases. As $\kappa$ increases, the real part of the real double roots increases relative to the real part of the complex pair of double roots so that a real instability determines the linear spreading speed for larger values of $\kappa$.

Absolute spreading speeds. One can easily verify that in the limiting regime $\gamma \sim 0$, absolute spreading speeds equal linear spreading speeds. In other words, the absolute spectrum is bounded to the right by a pair of double roots. To see this, we exploit continuity of the absolute spectrum in both $\gamma$ and $s$ to see that we need to control the absolute spectrum at $\gamma=$ 0, only. At this limiting point, one finds absolute spectrum from possibly four combinations of equations $\operatorname{Re}\left(\nu_{j}^{ \pm}-\nu_{m}^{ \pm}\right)=0$. The case $\operatorname{Re} \nu_{j}^{+}=\operatorname{Re} \nu_{j}^{-}$can be readily computed explicitly and gives a branch of absolute spectrum $\left\{\lambda \in \mathbb{R} ; \lambda \leq \lambda_{\mathrm{bp}}\right\}$. The combinations $\operatorname{Re} \nu_{j}^{-}=\operatorname{Re} \nu_{m}^{+}$, $m \neq j$ can be seen to be stable as follows. One first notices that the curves of absolute spectrum do not cross the imaginary axis. This is readily seen by substituting $\lambda=i \omega$ into the expressions (2.10) for $\nu_{j}^{ \pm}$and noticing that the real part of the roots is monotonically increasing with $\omega$. This excludes absolute spectrum in the right half plane for $s=s_{\text {lin }}$ since curves of absolute spectrum are connected to $\operatorname{Re} \lambda=-\infty$ in the complex plane [26]. One then verifies that the absolute spectrum close to the double roots is contained in the open left half plane: in fact, the curves of absolute spectrum are to leading order horizontal, for all values of $\kappa$. We will refer to this fact later, in Remark 4.13 and in Lemma 4.14, when we characterize pulled fronts.

\subsection{Onset of instability and Cahn-Hilliard spreading speeds}

A second limit in which analytic computations of spreading speeds are tractable is the regime near onset of the spinodal instability. We therefore investigate double roots in the parameter regime $f_{c}=1, f_{e}=d f_{c}+\mu$, with $\mu \geq 0$, small. We find

$$
d(\lambda-s \nu, \nu)=(\lambda-s \nu)^{2}+(1-\kappa)(\lambda-s \nu)-(1+\kappa) \nu^{2}(\lambda-s \nu)+\kappa \nu^{4}-\mu\left((\lambda-s \nu)-\nu^{2}\right),
$$

and

$$
\partial_{\nu} d(\lambda, \nu)=-2 \kappa(\lambda-s \nu)+\nu\left(4 \kappa \nu^{2}-2(1+\kappa)(\lambda-s \nu)+2 \mu\right)-(1-\kappa) s+s(1+\kappa) \nu^{2}+\mu s .
$$

We first discuss the case $s=0$. At $\mu=0$, double roots that satisfy the pinching condition are necessarily located in $\{\operatorname{Re} \lambda \leq 0\}$ since the essential spectrum is contained in the closed 
left half plane. We find $\lambda=\nu=0$ as the only critical double root at $\nu=0$. For $\mu>0$, one double root remains at $\lambda=0$ while two double roots cross into the unstable half plane at $\lambda=\mu^{2} / 4 \kappa+\mathrm{O}\left(\mu^{3}\right)$, with $\nu^{2}=-\mu /(2 \kappa)+\mathrm{O}\left(\mu^{2}\right)$. When increasing $s$, the real part of $\lambda$ associated with a double root decreases. We need to track the value of $s$ for which $\operatorname{Re} \lambda=0$. We therefore notice that we can solve (2.15)-(2.16) for $\lambda$ and $s$ as functions of $\nu$. The solutions can be expanded as

$$
\begin{gathered}
(1-\kappa) \lambda=3 \kappa \nu^{4}+\mu \nu^{2}+\mathrm{O}\left(|\nu|^{6}+\left|\mu \nu^{4}\right|+\left|\mu^{2} \nu^{2}\right|\right), \\
(1-\kappa) s=4 \kappa \nu^{3}+2 \mu \nu+\mathrm{O}\left(|\nu|^{5}+\left|\mu \nu^{3}\right|+\left|\mu^{2} \nu\right|\right) .
\end{gathered}
$$

Scaling

$$
\hat{\lambda}=\lambda(1-\kappa) \kappa / \mu^{2}, \quad \hat{s}=s(1-\kappa) \sqrt{\kappa} / \mu^{3 / 2}, \quad \hat{\nu}=\nu \sqrt{\kappa / \mu},
$$

gives

$$
\begin{aligned}
& \hat{\lambda}=3 \hat{\nu}^{4}+\hat{\nu}^{2}+\mathrm{O}(|\mu|), \\
& \hat{s}=4 \hat{\nu}^{3}+2 \hat{\nu}+\mathrm{O}(|\mu|) .
\end{aligned}
$$

At leading order, this system coincides with the linearization at a Cahn-Hilliard spinodal unstable equilibrium, and spreading speeds have been calculated in [35, §2.11 (82)]. We outline the calculation here in order to show that it is robust with respect to higher-order terms.

We obtain two real equations $\operatorname{Re} \hat{\lambda}=0, \operatorname{Im} \hat{s}=0$ for the complex variable $\nu$. The latter equation for $\hat{\nu}=\eta+\mathrm{i} k$ is at leading order

$$
4 \kappa k\left(3 \eta^{2}-k^{2}\right)+2 \mu k+k \mathrm{O}\left(\mu^{2}+|\mu|\left(\eta^{2}+k^{2}\right)+\eta^{4}+k^{4}\right)=0 .
$$

Dividing by the trivial double root at $k=0$, this gives $k^{2}=\mu / 2 \kappa+3 \eta^{2}+\mathrm{O}\left(\mu^{2}+|\mu| \eta^{2}+\eta^{4}\right)$ with the implicit function theorem. Substituting into $\operatorname{Re} \hat{\lambda}=0$, we obtain at leading order a quadratic equation for $\eta^{2}$ with one simple positive root. This yields expressions

$$
\eta^{2}=\frac{-1+\sqrt{7}}{24}+\mathrm{O}(\mu), \quad k^{2}=\frac{3+\sqrt{7}}{8}+\mathrm{O}(\mu)
$$

and a linear spreading speed and eigenvalue

$$
\hat{s}=\sqrt{\frac{2}{27}(17+7 \sqrt{7})}+\mathrm{O}(\mu), \quad \hat{\lambda}=\frac{\mathrm{i}(3+\sqrt{7})}{4} \sqrt{\frac{2+\sqrt{7}}{6}}+\mathrm{O}(\mu) .
$$

Summarizing, we find a unique linear spreading speed $s$ at the double root $(\lambda, \nu)$ with expan- 
sion in $\mu$

$$
\begin{aligned}
s_{\text {lin }} & =\frac{\mu^{3 / 2}}{(1-\kappa) \sqrt{\kappa}} \sqrt{\frac{2}{27}(17+7 \sqrt{7})}+\mathrm{O}\left(\mu^{5 / 2}\right), \\
\omega_{\text {lin }} & =\frac{\mu^{2}}{\kappa(1-\kappa)} \frac{3+\sqrt{7}}{4} \sqrt{\frac{2+\sqrt{7}}{6}}+\mathrm{O}\left(\mu^{3}\right) \\
\operatorname{Re} \nu_{\text {lin }} & =\sqrt{\frac{\mu}{\kappa}} \sqrt{\frac{-1+\sqrt{7}}{24}}+\mathrm{O}\left(\mu^{3 / 2}\right), \\
\operatorname{Im} \nu_{\text {lin }} & =\sqrt{\frac{\mu}{\kappa}} \sqrt{\frac{3+\sqrt{7}}{8}}+\mathrm{O}\left(\mu^{3 / 2}\right) .
\end{aligned}
$$

Again, in this case, one can verify that linear and absolute spreading speeds are equal. One finds that the absolute spectrum close to the imaginary axis is determined by the Cahn-Hilliard limiting problem. A somewhat lengthy calculation shows that double roots yield indeed the most unstable points in the absolute spectrum.

We finally note that in this regime as well as in the limit $f_{c} \sim 0$ (as well as in the Cahn-Hilliard equation itself), the frequencies in the comoving frame $\omega$ and in the steady frame $\omega-s \operatorname{Im} \nu$ do not vanish at leading order, so that the patterns generated in the wake of the front are not stationary in either steady or comoving frame. This phenomenon is illustrated in the righthand image of Figure 1.3, where near the leading edge of the invasion front, patterns appear to be slanted in the space-time plot, hence traveling and not stationary with $\omega-s \operatorname{Im} \nu \neq 0$.

\subsection{Numerical continuation of spreading speeds.}

We solved (2.4) numerically in the regime $\kappa<1 / 2$ using continuation from the limit $f_{c}=0$. We continued the complex branchpoint but did not check the pinching condition during the continuation procedure. We also did not check if other double roots crossed the imaginary during the continuation. We did however find good agreement with direct numerical simulations where comparisons were feasible. As expected, spreading speeds in direct numerical simulations were slightly lower than predicted [10,35].

We plotted results for several diffusion constants in Figure 2.1. The spreading speed is plotted against $f_{c}=\gamma$, setting $f_{e}=1-f_{c}$. Spreading speeds for different values of $f_{c}$ and $f_{e}$ are obtained by scaling $\left(f_{c}, f_{e}\right) \mapsto r^{2}\left(f_{c}, f_{e}\right)$ and $s \mapsto r s$.

We comment on some of these numerical results. First, note that there is a cross-over, so that spreading speeds increase with $\kappa$ for small $f_{c}$, the typical and expected behavior, but spreading speeds decrease when $\kappa$ is increased for larger values of $f_{c}$. This is in agreement with the predictions from (2.19), where $s_{\text {lin }}$ decreases when $\kappa \ll 1$ increases, and from (2.11), where $s_{\text {lin }}$ increases when $\kappa$ increases. Note however that the asymptotics in the Cahn-Hilliard limit $s \sim \mu^{3 / 2}$ are valid only in a very small range when $\kappa$ is small. Spreading speeds change little from $\kappa=0.01$ to $\kappa=0$.

The second plot in Figure 2.1 shows the selected wavenumber $k_{\text {lin }}=\omega_{\text {lin }} / s_{\text {lin }}$ as a function of $f_{c}$. We compared this selected wavenumber to $k_{\text {temp }}$ and $k_{\max }$, which are characteristics of the temporal dynamics rather than a spatial invasion process; see (1.8). As expected, 

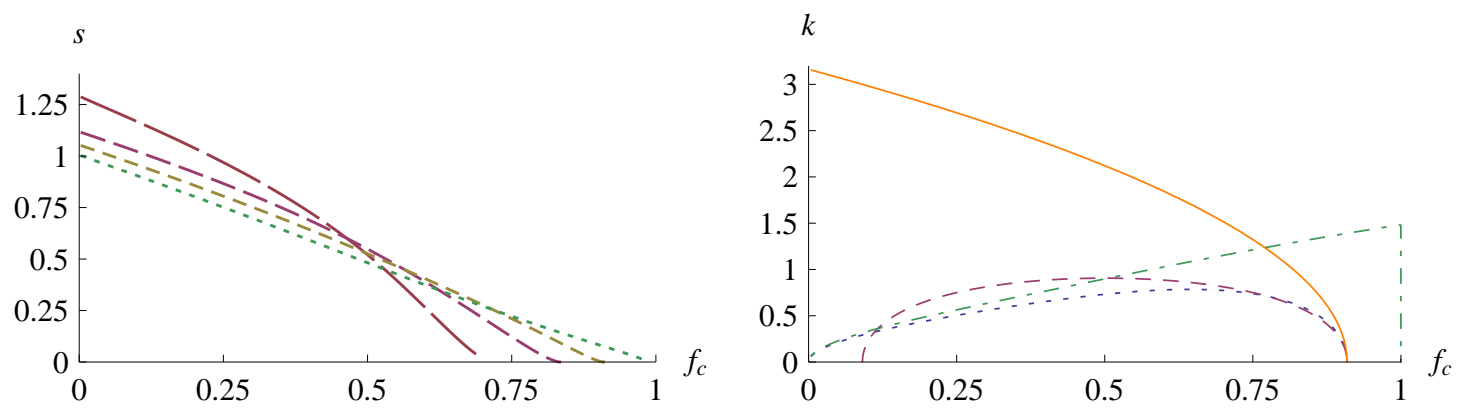

Figure 2.1: Spreading speeds and selected wavenumbers as functions of $f_{c}$, with $f_{e}=1-f_{c}$. On the left, spreading speeds for $\kappa=0.01,0.1,0.2,0.4$ as a function of $f_{c}$ (dash length increasing with $\kappa$ ). Spreading speeds are increasing in $\kappa$ for small $f_{c}$ and decreasing for larger values of $f_{c}$. Spreading speeds vanish at $f_{e}=\kappa f_{c}$, that is, $f_{c}=(1+\kappa)^{-1}$. On the right, a plot of wavenumbers $k_{\text {lin }}$ (dotted), $k_{\text {temp }}$ (dashed), and $k_{\max }$ (solid) over $f_{c}$ when $\kappa=0.1$; see text for definitions. We also included $k_{\text {lin }}$ for $\kappa=0$ (dotted-dashed); $k_{\mathrm{temp}}=k_{\max }=+\infty$ in this limit.

$k_{\max }>k_{\text {lin }}$ : a temporally unstable wavenumber is selected by the invasion process. The wavenumber selected by the invasion process is different from the wavenumber with fastest temporal growth rate, $k_{\text {lin }} \neq k_{\text {temp }}$. In particular, temporal dynamics select homogeneous states, $k_{\text {temp }}=0$, for small $f_{c}$, while invasion processes select patterns, $k_{\text {lin }}>0$.

Another remarkable fact is that even in the limit $\kappa=0$, where the linearization fails to select wavenumbers $\left(k_{\text {temp }}=k_{\max }=+\infty\right), 0<k_{\text {lin }}<+\infty$ : wavelengths are controlled by diffusion in the c-component, diffusion in the e-component is not crucial. This is in stark contrast with the expansions that we derived (for fixed $\kappa>0$ ), which show blowup of leading-order expansions of $k_{\text {lin }}$ in the weak coupling and the Cahn-Hilliard limit.

\section{Pushed fronts}

Linear spreading speeds give a lower bound on actual spread of disturbances: any spreading behavior with speed lower than the linear spreading speed would be linearly unstable (in the sense that perturbations grow pointwise, or that the absolute spectrum is unstable). A much more difficult question is to establish upper bounds. For this, one needs to control the effects of nonlinearity and, in particular, discuss the possible appearance of pushed fronts [35]. There can actually be multiple stable spreading mechanisms as illustrated in [2], where there are both a stable pulled front and a stable pushed front, defined in Section 3.1, below. In the simplest explicit example in [2], the two invasion mechanisms are distinguished by a topological winding number of the solution profile in the regime between leading edge and wake. All this said, we will not attempt to provide a complete picture of the invasion process, here, but focus on two aspects: in this section, we exhibit a regime where pushed fronts exist and create homogeneous states in their wake, and in the next section, we give a counting arguments for the existence of coherent pulled fronts.

The strategy in this section is to perturb from the uncoupled case, $\gamma=0$, where the system decouples into the well-understood scalar Nagumo-equation for $e$ and a linear equation for the temperature field $\theta$. We therefore start with a review of front propagation in the Nagumo 
equation in Section 3.2. In particular, we highlight a transition from pushed to pulled fronts. We then show how to obtain fronts in the full system using perturbative arguments in Section 3.3. For small diffusion constants $\kappa<1 / 2$, the coupling generates a new instability mechanism for pushed fronts, not present in the scalar Nagumo equation. In particular, pushed fronts exist in a much smaller regime for infinitesimally small coupling than in the absence of coupling.

Some of the analysis here is reminiscent of [6], where a similar limit has been studied. As pointed out in Remark 1.1, our situation is different since we are interested in fronts connecting two unstable states.

\subsection{Pushed fronts and strong decay}

Many definitions of pushed front propagation assume in some way that the system is scalar. We give a more general definition, here. Consider therefore the general reaction-diffusion system (2.1) with dispersion relation $d(\lambda, \nu)$ for the linearization near an unstable state $u=-u_{+}$. For $s>s_{\text {abs }}$, the absolute spectrum does not contain points $\lambda \geq 0$. We can therefore order roots $\nu$ of $d_{s}(0, \nu)$ with increasing real part $\operatorname{Re} \nu_{1} \leq \ldots \leq \operatorname{Re} \nu_{N}<\operatorname{Re} \nu_{N+1} \leq \ldots<\operatorname{Re} \nu_{2 N}$.

We say that a traveling wave $u(x-s t)$ is a pushed front if $\left|u(\xi)-u_{+}\right| \leq C \mathrm{e}^{\left(\operatorname{Re} \nu_{N}+\delta\right) \xi}$, for all $\xi \geq 0$, for any $\delta>0$ and some $C=C(\delta)>0$.

In many cases, $\nu_{N}<\nu_{N+1}<0$, and $\nu_{N}<\nu_{N+1}^{*}<0$, where $\nu_{N+1}^{*}$ is computed for $\lambda=0$ and $s=s_{\text {lin }}$. In other words, pushed fronts, as defined here, often decay faster than pulled fronts.

We will see later that pushed fronts typically occur in a robust fashion, for specific values of the wave speed $s$; see Section 4.7. Without going into details, we note that our definition here guarantees precisely that $\lambda=0$ belongs to the extended point spectrum of the front: in appropriately weighted spaces, the linearization at the front is Fredholm with index 0 and possesses a one-dimensional kernel associated with translations of the front. This is in general not true for pulled fronts or other fronts (without strong decay) with $s \neq s_{\text {lin }}$.

\subsection{Pushed and pulled fronts in the Nagumo equation}

We review front propagation in the Nagumo equation,

$$
e_{t}=\kappa e_{x x}+e(1-e)(e-a)
$$

with $0<a<1 / 2$. We are interested in front solutions $e(x-s t)$ connecting $e=1$ at $x=-\infty$ to $e=a$ at $x=+\infty$. Such fronts solve the traveling-wave ODE

$$
e_{\xi}=w, \quad w_{\xi}=-\kappa^{-1}(s w+e(1-e)(e-a)) .
$$

The dispersion relation $d(\lambda, \nu)=\kappa \nu^{2}+s \nu+a(1-a)-\lambda$ possesses roots $\nu_{N}<\nu_{N+1}<0$ at $\lambda=0$ for $s>s_{\text {lin }}$. Pushed fronts correspond to heteroclinic orbits that lie in the strong stable manifold of $e=a, w=0$.

For $a>1 / 3$, such heteroclinic orbits do not exist. In other words, $s_{\mathrm{nl}}=s_{\text {lin }}$, the linear spreading speed, which is given by $s_{\text {lin }}=2 \sqrt{\kappa a(1-a)}$. In the traveling-wave ODE, this linear spreading speed is characterized by a double eigenvalue at the equilibrium $e=a$. This double eigenvalue corresponds, of course, to a double root at $\lambda=0$ of the linear dispersion 

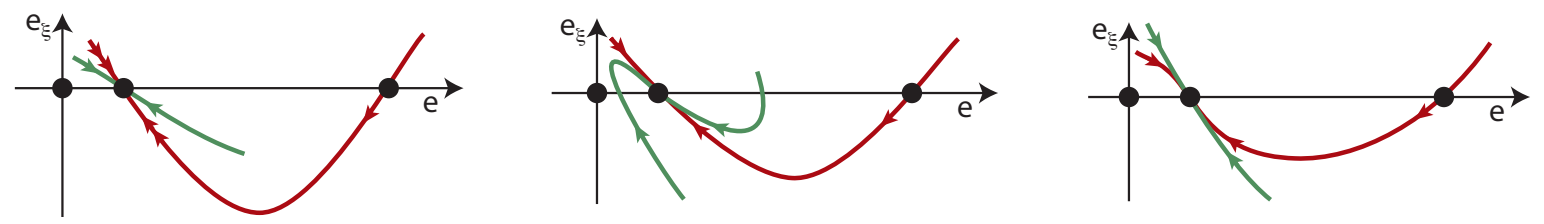

Figure 3.1: Phase portraits for (3.1) for $a<1 / 3$, left, $a=1 / 3$, middle, and $a>1 / 3$, right. The speed parameter $s$ is chosen to be the pushed (left) and pulled (right) front speed, respectively. Both speeds agree at $a=1 / 3$.

relation. Since there are only two roots $\nu$ of the dispersion relation, the double root that characterizes the linear spreading speed automatically satisfies the pinching condition in the Nagumo system. In particular, crossing of this double root induced by decreasing $s$ induces an absolute instability in the comoving frame. For speeds below the linear spreading speed, fronts possess oscillatory decay towards $e=a$.

For $a<1 / 3$, heteroclinic orbits that connect to the strong stable manifold exist, so that $s_{\mathrm{nl}}>s_{\text {lin }}$. Shape and speed of the pushed front can be computed explicitly in this particular cubic case,

$$
e_{\text {pushed }}(\xi)=\frac{a+\mathrm{e}^{\nu_{\mathrm{ss}} \xi}}{1+\mathrm{e}^{\nu_{\mathrm{ss}} \xi}}, \quad \xi=x-s_{\mathrm{nl}} t, \quad s_{\mathrm{nl}}=\frac{(a+1) \sqrt{2 \kappa}}{2}, \quad \nu_{\mathrm{ss}}=-\frac{1-a}{\sqrt{2 \kappa}} .
$$

For speeds just below $s_{\mathrm{nl}}$, fronts are non-monotone, with a unique minimum less than $a$.

Figure 3.1 illustrates the transition from $a<1 / 3$ to $a>1 / 3$ in the phase portrait of (3.1).

In this scalar case, one can actually show that fronts are stable in an appropriately weighted space if and only if they are monotone. In particular, pushed and pulled fronts are stable, and $s_{\mathrm{nl}}$ happens to be the smallest speed so that there exists a monotone front solution.

\subsection{Pushed fronts in the system with small $\gamma$}

Given a front in the Nagumo equation, we aim to find a nearby front to the system for small values of $\gamma$ using perturbation analysis. Writing out the traveling-wave equation for $(c, e)(x-s t)$, substituting $\theta=c+\kappa e$ for $c$, and integrating the $\theta$-equation once, one finds

$$
\begin{aligned}
e_{\xi} & =w \\
w_{\xi} & =-\kappa^{-1}(s w+e(1-e)(e-a)+\gamma(\theta-\kappa e)) \\
\theta_{\xi} & =-s(\theta+(1-\kappa) e-m) .
\end{aligned}
$$

Here, the mass $m$ arises as a constant of integration in the $\theta$-equation. Notably, the mass $c+e=\theta+(1-\kappa) e$ of any equilibrium is given by $m$, the integration constant, which confirms the intuitive claim that traveling waves preserve mass.

In the following, we fix $m=a$, so that $e=a, \theta=\kappa a$, and $e=1+\mathrm{O}(\gamma), \theta=a+\kappa-1+\mathrm{O}(\gamma)$ are equilibria with $w=0$. We refer to these equilibria as $E_{a}$ and $E_{1}$, respectively. Linearizing at those equilibria and using that $s>0$, we find that $E_{a}$ is asymptotically stable and $E_{1}$ is hyperbolic with one-dimensional unstable manifold, which we denote by $W^{\mathrm{u}}(1)$. At $\gamma=0$, 
the eigenvalues of the linearization at $E_{a}$ are given by $\nu_{\mathrm{ss}}<\nu_{\mathrm{s}}<0$, and $-s$. Here, the $\nu_{j}$ solve $\kappa \nu_{j}^{2}+s \nu_{j}+a(1-a)=0$. The following lemma gives the crucial link between the value of $a$ and the ordering of eigenvalues.

Lemma 3.1 Set $s=(a+1) \sqrt{\kappa / 2}$, the speed of the pushed Nagumo front (3.2). Then $a<$ $a_{*}(\kappa)$ is equivalent to $-s<\nu_{\mathrm{s}}$.

Proof. The proof is a direct calculation.

In the regime $a<a_{*}(\kappa)$, the equilibrium $E_{a}$ therefore possesses a two-dimensional strong stable manifold $W^{\mathrm{ss}}(a)$, tangent to the eigenspaces corresponding to eigenvalues $\nu_{\mathrm{ss}}$ and $-s$, for all $\gamma$ small enough. We will see below that the Nagumo front induces a decay $\mathrm{e}^{-s \xi}$ in the $\theta$-component, so that the Nagumo pushed fronts are pushed fronts for the system only when $a<a_{*}$ in the limit $\gamma=0$.

Lemma 3.2 For $0<a<a_{*}(\kappa)$ and $m \in \mathbb{R}$ fixed, there exists $\gamma_{0}>0$ such that for all $0 \leq \gamma<\gamma_{0}$, there is a pushed front, that is, a traveling wave solution in the intersection $W^{\mathrm{u}}(1) \cap W^{\mathrm{ss}}(a)$ for a speed $s=s(m, \gamma, a)$. The traveling wave and the speed depend smoothly on the parameters $m, \gamma, a$.

Proof. First notice that for $\gamma=0$, there exists a pushed front of the required form in the $e-w$-subsystem. One readily checks that the unique bounded solution to the equation for $\theta$ is given through

$$
\theta(\xi)=-s \mathrm{e}^{-s \xi} \int_{-\infty}^{\xi} \mathrm{e}^{s \tau}((1-\kappa) e(\tau)-m) \mathrm{d} \tau=-s(1-\kappa) \int_{0}^{\infty} \mathrm{e}^{-s \sigma} e(\xi-\sigma) \mathrm{d} \sigma+m<\infty
$$

Since $e$ converges exponentially as $\xi \rightarrow \infty$, one finds that $\theta$ converges exponentially. Since moreover $e$ is monotone, it solves a scalar equation $e_{\xi}=\tilde{h}(e)$. Together with the equation for $\theta$ and the linearization at the asymptotic states, one finds that $\theta$ converges with rate $\mathrm{e}^{-s \xi}$ as $\xi \rightarrow+\infty$, so that the corresponding heteroclinic is indeed contained in the strong stable manifold of $e=a$. The roots of the dispersion relation are given by $\nu_{\mathrm{ss}}, \nu_{s},-s$, and $\nu=0$. The last root is induced by the conserved quantity and was eliminated from the traveling-wave equation by integrating the $\theta$-equation. Since $\max \left(\nu_{\mathrm{ss}},-s\right)<\min \left(\nu_{\mathrm{s}}, 0\right)$, solutions in the strong stable manifold of our traveling-wave systems are indeed pushed fronts according to the definition in Section 3.1.

It remains to prove that this intersection is transverse in $s$. To see this, consider the linearization at the heteroclinic, which is a Fredholm operator of index 0 , with one-dimensional kernel in $B C^{0}\left(\mathbb{R}, \mathbb{R}^{3}\right)$, say. Due to the block-triangular structure of the system at $\gamma=0$, the kernel of the adjoint is supported in $e-w$-space, only, that is, the $\theta$-component vanishes. The $e-w$-component is in fact given by the kernel of the adjoint to the $e-w$-equation, only. We conclude that the derivative of the system with respect to $s$ is not contained in the range of the linearization with respect to $(\theta, e, w)$ since this is not the case for the $e-w$ subsystem. 
In consequence, the intersection is transverse and the heteroclinic persists, smoothly depending on system parameters with values in $B C^{0}\left(\mathbb{R}, \mathbb{R}^{3}\right)$, and, by a simple boot strap, also in $B C^{k}\left(\mathbb{R}, \mathbb{R}^{3}\right)$ for any $k$.

The heteroclinic orbits found in the previous lemma are in fact spectrally stable pushed fronts. Therefore, define the exponentially weighted spaces $L_{\eta_{ \pm}}^{2}$,

$$
\|u\|_{\eta_{ \pm}}^{2}=\int_{\xi>0}\left|u(\xi) \mathrm{e}^{-\eta_{+} \xi}\right|^{2} \mathrm{~d} \xi+\int_{\xi<0}\left|u(\xi) \mathrm{e}^{-\eta_{-} \xi}\right|^{2} \mathrm{~d} \xi
$$

We will choose weights $\eta_{ \pm}$so that the essential spectrum of the linearization is contained in the open left half plane. More precisely, we choose

$$
\eta_{+} \in\left(\max \left\{-s, \nu_{\mathrm{ss}}\right\}, \nu_{\mathrm{s}}\right), \quad \eta_{-} \in(-s, 0),
$$

where we used the values

$$
\nu_{\mathrm{ss}}=-\frac{1-a}{\sqrt{2 \kappa}}<\nu_{\mathrm{s}}=-\frac{2 a}{\sqrt{2 \kappa}} .
$$

Lemma 3.3 The fronts constructed in Lemma 3.2 are spectrally stable in $L_{\eta_{-}, \eta_{+}}^{2}$ with $\eta_{ \pm}$ chosen as in (3.5), that is, the spectrum of the linearization is contained in the open left half plane with the exception of an algebraically simple isolated eigenvalue located at the origin.

Proof. We start by locating the spectrum at $\gamma=0$. Because of the tridiagonal form, it is sufficient to locate the spectrum of the diagonal parts, $L_{\theta}$ and $L_{e}$, where

$$
L_{\theta} \theta=\theta_{x x}+s \theta_{x}, \quad L_{e} e=\kappa e_{x x}+s e_{x}+g^{\prime}\left(e_{*}\right) e .
$$

One readily verifies that the constant coefficient operator $L_{\theta}-\lambda$ is invertible with the given choice of weights in $\operatorname{Re} \lambda \geq 0$. The essential spectrum of the operator $L_{e}$ is contained in the open left half plane with the exception of an algebraically simple zero eigenvalue. As a consequence, the assertion of the lemma is true for $\gamma=0$.

Since the front itself and thereby the operators depend smoothly on $\gamma$, the spectrum changes continuously with $\gamma$. This shows stability in the exponentially weighted spaces up to a possible eigenvalue near the origin. Since the eigenvalue $\lambda=0$ is algebraically simple at $\gamma=0$, there is a unique eigenvalue in a neighborhood of the origin for all $\gamma$ close to zero. The derivative of the front itself provides us with a candidate for an eigenfunction to a zero eigenvalue. We need to show that this derivative actually belongs to $L_{\eta_{-}, \eta_{+}}^{2}$. Since the front lies in $W^{\mathrm{u}}(a) \cap W^{\mathrm{ss}}(1)$, the derivative belongs to the intersection of the tangent spaces. In particular, the derivative is bounded as $\xi \rightarrow-\infty$ and decays with exponential rate $\mathrm{e}^{\eta \xi}, \eta \leq \max \left\{\nu_{\mathrm{ss}},-s\right\}$. With our choice of exponential weights, the derivative therefore lies in $L_{\eta_{-}, \eta_{+}}^{2}$. This shows that the zero eigenvalue is pinned at the origin for all $\gamma$ and proofs the lemma.

Lemma 3.4 For $a>a_{*}(\kappa)$, pushed fronts do not exist in the limit $\gamma=0$. 
Proof. For $\kappa<1 / 2, a>a_{*}(\kappa)$, the strong stable manifold is tangent to eigenspaces associated with $\nu_{\mathrm{ss}}$ and $\nu_{\mathrm{s}}$, since $\nu_{\mathrm{s}}<-s$. However, (3.4) does not possess solutions with decay stronger than $\mathrm{e}^{-s \xi}$. This can be readily seen by substituting the asymptotics $e \sim e_{\infty} \mathrm{e}^{\nu_{s} \xi}$ into (3.4) and expanding the integral in $\mathrm{e}^{-s \xi}$.

In the case $\kappa>1 / 2, a>a_{*}(\kappa)=1 / 3$, fronts in the Nagumo equation decay with rate $\mathrm{e}^{\nu_{\mathrm{s}} \xi}$. For monotone fronts, one again finds from (3.4) that $\theta$ decays with minimal rate $\mathrm{e}^{\nu \xi}$, $\nu=\max \left\{-\nu_{\mathrm{s}},-s\right\}$. This proves the lemma.

We next exclude pushed fronts for small $\gamma>0$.

Proposition 3.5 Fix $\kappa>0$. For any $\delta>0$ there is $\gamma_{0}>0$ so that there are no pushed fronts that connect $e=1+\mathrm{O}(\gamma)$ to $c=0, e=a$ for $1 / 2 \geq a>a_{*}(\kappa)+\delta$ and $0 \leq \gamma<\gamma_{0}$.

Proof. Pushed fronts lie in the intersection of the strong stable manifold of $e=a, \theta=\kappa a$ and the unstable manifold of another equilibrium. Suppose that we would have a sequence of pushed fronts with $\gamma_{k} \rightarrow 0$. Since one can easily exclude pushed fronts for large speeds, we can assume that $s_{k} \rightarrow s$ and $a_{k} \rightarrow a_{0}>a_{*}(\kappa)$. We will show below that the associated sequence of heteroclinic orbits is bounded. Continuity of the strong stable manifold then shows that an appropriately shifted subsequence converges locally uniformly to a bounded trajectory in the strong stable manifold of $e=a, \theta=\kappa a$ in the limit. This trajectory would correspond to a pushed front in the limit, which however was excluded in Lemma 3.4.

It remains to show that the set of bounded solutions to (3.3) is bounded, uniformly in $\gamma_{0}>\gamma \geq$ 0 , and $m \in\left[m_{-}, m_{+}\right]$. This follows readily from the maximum principle as follows. Applied to the second-order equation for $e$, the maximum principle gives $\sup |e(\xi)| \leq c_{1} \sup |\theta(\xi)|^{1 / 3}+c_{2}$. From the equation for $\theta$, we find $\sup |\theta(\xi)| \leq c_{3} \sup |e(\xi)|+c_{4}$. Combining these two, we find that both $e$ and $\theta$ are uniformly bounded as claimed.

\section{Transient versus persistent pattern forming fronts}

In the previous section, we have seen that for $a<a_{*}(\kappa)$, fronts create a bulk state in their wake. For larger $a$, front propagation is typically oscillatory. Our goal here is to give crude counting arguments for the existence of oscillatory fronts. Our main results give criteria for robustness of fronts as stated in Theorem 2 .

We discuss the problem of finding traveling-wave solutions that describe the invasion problem near an unstable equilibrium. We therefore look for solutions to the reaction-diffusion system in a frame moving with the linear spreading-speed $s=s_{\text {lin }}$.

Our linear analysis from Section 2 predicts that in this comoving frame $\xi=x-s t$, solutions will resemble exponentials $\mathrm{e}^{\lambda t+\nu \xi}$, with $\lambda=\mathrm{i} \omega_{\text {lin }}$ and $\nu=\nu_{\text {lin }}$, the solutions to $d_{s}(\lambda, \nu)=$ $\partial_{\nu} d_{s}(\lambda, \nu)=0$. Since $\omega_{\text {lin }} \neq 0$ for almost all $\gamma$, such solutions are not stationary in the comoving frame but necessarily time-periodic, with frequency $\omega_{\text {lin }}$. We therefore look for solutions to the reaction-diffusion system that are time-periodic in the frame $\xi$, with period $T=2 \pi / \omega_{\text {lin }}$. We refer to such periodic solutions, with exponential decay at $\xi=+\infty$ as described above, as coherent invasion fronts. We emphasize that nonlinear effects may well 
create subharmonic coherent fronts, with temporal periods $\ell T, 1<\ell \in \mathbb{Z}$. Most of our analysis can be adapted to this case.

While the linear analysis predicts the growth of spatio-temporal oscillations, it is not clear if this linear growth will eventually create a periodic pattern. In fact, all periodic patterns are unstable as solutions on the real line; see Lemma 4.18, below. One may argue that the invasion of an unstable state should "typically" generate a stable pattern, so that fronts that leave a periodic pattern in their wake would be untypical. We will see however that this intuitive reasoning needs to be refined.

Our strategy involves dimension counting similar to arguments involved in the study of defects in oscillatory media [29]; see also [30]. We view coherent fronts as heteroclinic orbits and count dimensions of stable and unstable manifolds. The key argument involves a homotopy of stable and unstable eigenspaces during which changes in dimension can be related to resonant unstable modes. Since this analysis is more widely applicable, we present the main definitions and tools in a general setting of reaction-diffusion systems, before specializing to our specific kinetics.

Outline: We first present counting arguments for traveling fronts in Section 4.1. The basic idea is to define robustness in terms of dimensions of stable and unstable manifolds in the traveling-wave equation. We motivate the arguments in the case $D=$ id in Section 4.1.1, first. We then present the basic homotopy argument in Section 4.1.2 and relate multiplicities to group velocities in Section 4.1.3. We then generalize these concepts to modulated fronts, which are time-periodic in a moving frame of reference. We motivate modulated fronts and interpret them as heteroclinic orbits in Section 4.2 and introduce the basic definition of robustness in Section 4.3. We outline the strategy for the computation of dimensions of stable and unstable manifolds in the modulated traveling-wave equation in Sections 4.4 and 4.5, for spatially homogeneous and for spatially periodic equilibria, respectively. Section 4.6 introduces the concept of critical decay, slightly restricting the class of pulled fronts, and Section 4.7 briefly digresses to counting arguments for pushed fronts. Finally, in Section 4.8, we apply these concepts to our case of precipitation kinetics and prove our main result, Proposition 4.23, from which Theorem 2 follows as a special case.

\subsection{Counting traveling fronts}

Consider a reaction-diffusion system

$$
u_{t}=D u_{x x}+F(u) \in \mathbb{R}^{N},
$$

with $D+D^{*}>0$ and equilibria $F\left(u_{ \pm}\right)=0$. We are interested in the traveling-wave equation

$$
\begin{aligned}
& u_{\xi}=v \\
& v_{\xi}=-D^{-1}(s v+F(u)),
\end{aligned}
$$

which we write in the short form

$$
w_{\xi}=A w+G(w)
$$


with

$$
A=\left(\begin{array}{cc}
0 & 1 \\
0 & -D^{-1} s
\end{array}\right), \quad G(w)=\left(\begin{array}{c}
0 \\
-D^{-1} F(u)
\end{array}\right) .
$$

This traveling-wave equation possesses the equilibria $w_{ \pm}=\left(u_{ \pm}, 0\right)$. We are interested in heteroclinic orbits connecting these two equilibria and would therefore like to determine dimensions of stable and unstable manifolds. The key observation is that temporal stability information from (4.1) determines the dimension of the stable manifold of $w_{+}$in (4.2). The connection is provided by the dispersion relation, which determines temporal stability. In fact, eigenvalues $\nu$ of the linearization at the equilibrium $(u, v)=\left(u_{+}, 0\right)$ solve $d_{s}(0, \nu)=\operatorname{det}\left(D \nu^{2}+s \nu+F^{\prime}\left(u_{+}\right)\right)=0$.

\subsubsection{The case $D=\mathrm{id}$.}

For $D=\mathrm{id}$, one can find eigenvalues $\nu$ from eigenvalues $\lambda_{j}$ of $F^{\prime}\left(u_{+}\right)$by solving $\nu^{2}+s \nu+\lambda_{j}=0$, with solutions $\nu_{j}^{ \pm}$. Recall that throughout we assume $s>0$. One then sees that $\pm \operatorname{Re} \nu_{j}^{ \pm}>0$ when $\operatorname{Re} \lambda_{j}<\left(\operatorname{Im} \lambda_{j} / s\right)^{2}$, and that $\operatorname{Re} \nu_{j}^{ \pm}<0$ when $\operatorname{Re} \lambda_{j}>\left(\operatorname{Im} \lambda_{j} / s\right)^{2}$. In other words, a simple eigenvalue $\nu$ crosses into the left half plane when $\lambda_{j}$ increases across the curve $\operatorname{Re} \lambda=$ $(\operatorname{Im} \lambda / s)^{2}$. If we assume that the spectrum of $F^{\prime}\left(u_{+}\right)$consists of eigenvalues $\lambda_{1}>0>\operatorname{Re} \lambda_{j}$, $j \leq 2 \leq N$, there are precisely $N-1$ spatial eigenvalues $\nu$ with positive real part and $N+1$ spatial eigenvalues with negative real part in the traveling-wave equation.

If on the other hand we assume that $u_{-}$is stable, that is, $\operatorname{Re} \lambda_{j}<0$ for all $\lambda_{j}$ in the spectrum of $F^{\prime}\left(u_{-}\right)$, we find that the linearization at $w_{-}=\left(u_{-}, 0\right)$ possesses precisely $N$ stable and $N$ unstable eigenvalues $\nu$. As a consequence, intersections between the $N$-dimensional unstable manifold of $w_{-}$and the $N+1$-dimensional stable manifold of $w_{+}$can be transverse, along the necessarily at least one-dimensional intersection given by an orbit $w(\xi)$. We then say that the traveling wave is robust by dimension counting. In particular, a traveling wave traveling at the linear spreading speed can be given by a robust heteroclinic orbit in this case. We refer to Definition 4.4 for a formal definition of robustness.

On the other hand, if $u_{-}$is temporally unstable with respect to a simple real eigenvalue, too, then the unstable manifold of $w_{-}$in the traveling-wave equation is $N$-1-dimensional, and intersections with the $N+1$-dimensional stable manifold of $w_{+}$cannot be transverse for a fixed wave speed $s$. We conclude that invasion at the linear spreading speed will typically not select an unstable state $u_{-}$.

Interestingly, when we assume that $u_{-}$is hyperbolic, unstable precisely with respect to a complex conjugate pair of eigenvalues $\{\lambda, \bar{\lambda}\}$, with large enough imaginary part $\operatorname{Re} \lambda<(\operatorname{Im} \lambda / s)^{2}$, we find that two of the associated roots $\nu$ possess positive real part and two possess negative real part. As a consequence, the dimension counting argument predicts that at the linear spreading speed one can have a robust traveling wave that leaves behind an unstable state provided the instability is oscillatory with large enough frequency.

\subsubsection{The general case $-\lambda$-homotopies.}

The reasoning above was oversimplified since we assumed that $D$ was a scalar, which allowed us to make the connection between spatial rates $\nu$ and temporal rates $\lambda$ explicit. Slightly 
generalizing, our arguments apply whenever $D$ and $F^{\prime}$ are simultaneously diagonalizable. Unfortunately, this still excludes most interesting cases, for instance the case of precipitation kinetics (1.3) which is of interest here.

In the general case when $D$ is a positive matrix, for instance $D=\operatorname{diag}\left(d_{j}\right)>0$, it is convenient to count dimensions using a homotopy argument.

We introduce some convenient terminology, first. We refer to the number of unstable eigenvalues $(\operatorname{Re} \nu>0)$ of the linearization at an equilibrium of the traveling-wave equation, counted with multiplicity, as the Morse index $i$. We call $i-N$ the relative Morse index. As we saw above, stable equilibria have relative Morse index 0 in the case $D=$ id. Equilibria that are unstable with respect to one real unstable eigenvalue possess relative Morse index -1 . Connecting orbits where the (relative) Morse index decreases by one from $-\infty$ to $+\infty$ are robust by dimension counting.

Recall that the dispersion relation is obtained through the ansatz $u \sim \mathrm{e}^{\lambda t+\nu x}, d(\lambda, \nu)=$ $\operatorname{det}\left(D \nu^{2}+F^{\prime}-\lambda\right)$, where $F^{\prime}$ is evaluated at either $u_{+}$or $u_{-}$. In the comoving frame, the dispersion relation is $d_{s}(\lambda, \nu)=d(\lambda-s \nu, \nu)$. Also, recall that $\lambda$ is in the essential spectrum when $d(\lambda, \mathrm{i} k)=0$ for some $k \in \mathbb{R}$. Eigenvalues $\nu$ of the traveling-wave equation are roots of $d_{s}(0, \nu)$, counted with multiplicity. The main idea is to count roots of $d_{s}(0, \nu)=0$ in $\operatorname{Re} \nu>0$ by tracking how roots cross $i \mathbb{R}$ during a homotopy from $\lambda=+\infty$ to $\lambda=0$. At $\lambda \sim+\infty$, one easily finds that roots solve $d_{j} \nu^{2}=\lambda$ at leading order, so that there are precisely $N$ roots with positive real part - the relative Morse index is 0 .

In order to determine the relative Morse index at $\lambda=0$, we need to locate values of $\lambda$ during the homotopy where the relative Morse index changes. This happens precisely when spatial eigenvalues are located on the imaginary axis, that is, when $d_{s}(\lambda, \mathrm{i} k)=0$ for some $k \in \mathbb{R}$, that is, on curves of essential spectrum. This motivates the following definition.

Definition 4.1 (Resonant modes - traveling waves) We say that the wavenumber $k$ corresponds to a resonant neutral mode $(0, \mathrm{i} k)$ if $d_{s}(0, \mathrm{i} k)=d(-s \mathrm{i} k, \mathrm{i} k)=0$. Similarly, we define resonant growth modes $(\lambda, \mathrm{i} k) \in \mathbb{R}_{+} \times \mathrm{i} \mathbb{R}$ through the condition $d_{s}(\lambda, \mathrm{i} k)=0$. In both cases, we call $k$ a resonant spatial wavenumber.

We say that a resonant growth mode $(\lambda, \mathrm{i} k)$ is temporally simple when $\partial_{\lambda} d_{s}(\lambda, \mathrm{i} k) \neq 0$.

We define the spatial multiplicity of resonant modes $(\lambda, \mathrm{i} k)$ as the sum of the orders of the root $\nu=\mathrm{i} k$ of $d_{s}(\lambda, \cdot)$. The total spatial multiplicity of $\lambda$ is the sum over all multiplicities of resonant spatial wavenumbers associated with $\lambda$.

The concept of resonance will become somewhat clearer later when we study modulated traveling waves. For now, resonance refers to temporal behavior of a mode that is in resonance with the temporal behavior of the wave: since the traveling waves under consideration are stationary in a comoving frame, we require resonant modes to be stationary (or to possess real exponential growth) in a comoving frame. 


\subsubsection{Crossing numbers}

With any simple resonant mode, we can associate a generalized group velocity $s_{\mathrm{g}}=-\operatorname{Re} \frac{\mathrm{d} \lambda}{\mathrm{d} \nu}=$ $\operatorname{Re}\left(\partial_{\nu} d_{s} / \partial_{\lambda} d_{s}\right)$. The term group velocity is justified by the fact that for $\lambda=\mathrm{i} \omega, \nu=-\mathrm{i} k \in \mathrm{i} \mathbb{R}$, the expression reduces to the classical expression $s_{\mathrm{g}}=\frac{\mathrm{d} \omega}{\mathrm{d} k}$. Since $\partial_{\nu} d_{s}=-s \partial_{\lambda} d+\partial_{\nu} d$, we find the typical Galilei transformation of group velocities $s_{\mathrm{g}}^{\mathrm{co}}$ in the comoving and $s_{\mathrm{g}}^{\text {st }}$ in the steady frame $(s=0), s_{\mathrm{g}}^{\mathrm{co}}=s_{\mathrm{g}}^{\mathrm{st}}-s$.

Lemma 4.2 Assume that neutral and growing modes are simple and have nonvanishing group velocities $s_{\mathrm{g}}$ in the comoving frame. Denote by $m_{ \pm}$the total multiplicities corresponding to positive and negative group velocities. Then the relative Morse index of $u$ in the traveling-wave equation is $m_{+}-m_{-}$.

Proof. We follow the relative Morse index in a homotopy from $\lambda=+\infty$ to $\lambda=0$. At $\lambda=+\infty$, the relative Morse index is zero. The relative Morse index can change only when $\lambda=\lambda_{*}$, one of finitely many resonant growth modes. For such a $\lambda_{*}$, there are $m_{*}<\infty$ roots $\nu_{j}$ on the imaginary axis. Since we assumed that the resonant modes were simple, $\partial_{\lambda} d_{s}\left(\lambda_{*}, \nu_{j}\right) \neq 0$, one readily computes that

$$
\operatorname{sign} \frac{\mathrm{d} \operatorname{Re} \nu}{\mathrm{d} \lambda}=-\operatorname{sign} \operatorname{Re} \frac{\partial_{\lambda} d_{s}}{\partial_{\nu} d_{s}}=-\operatorname{sign} \operatorname{Re} \frac{\partial_{\nu} d_{s}}{\partial_{\lambda} d_{s}}=-\operatorname{sign} s_{\mathrm{g}} .
$$

As a consequence, the relative Morse index is reduced by the total multiplicity $\ell_{-}$of the

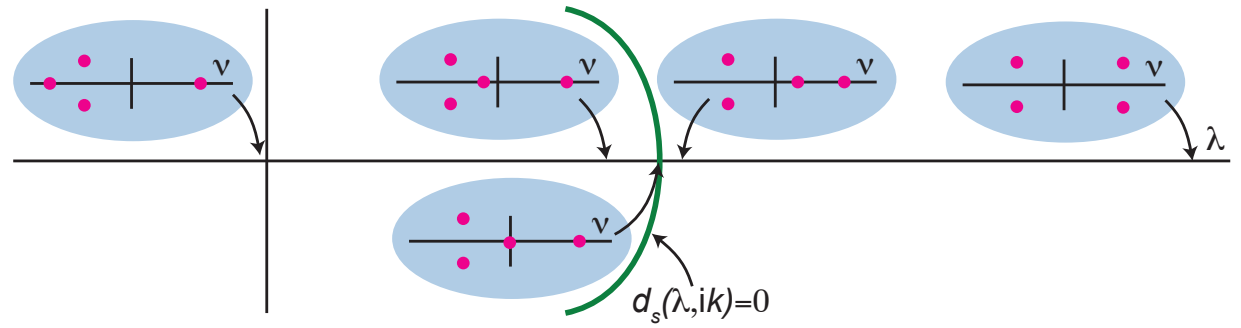

Figure 4.1: The figure illustrates the change of the relative Morse index from 0 to -1 as $\lambda$ is decreased from $\lambda=+\infty$ to $\lambda=0$, crossing a curve of resonant modes $d_{s}(\lambda, \mathrm{i} k)=0$. Shown is the complex plane $\lambda \in \mathbb{C}$ with insets representing the locations of roots $\nu \in \mathbb{C}$ to $d_{s}(\lambda, \cdot)=0$ depending on $\lambda \in \mathbb{C}$. Lemma 4.2 relates the direction of crossing to group velocities.

resonant growth modes with negative group velocities and increased by the total multiplicity $\ell_{+}$of the resonant growth modes with positive group velocities when $\lambda$ is decreased through all resonant growth modes; see also Figure 4.1 for an illustration. A similar argument applies to neutral modes. For instance, neutral modes with negative group velocity satisfy $\partial_{\lambda} \operatorname{Re} \nu>0$. They therefore lie in $\operatorname{Re} \nu>0$ for $\lambda>0$ and hence reduce the Morse index by their total multiplicity, since the Morse index only counts roots with $\operatorname{Re} \nu>0$.

Remark 4.3 (Multiple resonant modes) One can define and compute crossing numbers of resonant modes also in the case when $\partial_{\lambda} d_{s}(\lambda, \mathrm{i} k)=0$ or when $c_{\mathrm{g}}=0$. Of course, crossing 
numbers are implicitly defined through the change in Morse index, which is equivalent to the signed number of roots $\nu$ that cross the imaginary axis as $\lambda$ is decreased. Somewhat more explicitly, one can approximate $d_{s}^{\varepsilon}:=d_{s}+\varepsilon, \varepsilon \in \mathbb{C}$. Viewing $d_{s}^{\varepsilon}=0$ as two real equations in the real variables $\lambda \in \mathbb{R}$ and $k=\operatorname{Im} \nu \in \mathbb{R}$, Sard's theorem gives us a sequence of $\varepsilon_{j} \rightarrow 0$ so that both $\partial_{\lambda} d_{s}^{\varepsilon}$ and $\partial_{k} d_{s}^{\varepsilon}$ do not vanish at roots $d_{s}^{\varepsilon}=0$. In particular, all resonant modes are simple and group velocities do not vanish. Since Morse indices stabilize as $\varepsilon_{j} \rightarrow 0$ outside of resonant modes, this allows us to compute relative Morse indices from group velocities for the perturbed dispersion relation, provided that $\varepsilon_{j}$ is sufficiently small.

\subsection{Modulated fronts as a dynamical system}

As pointed out in the beginning of this section, we are interested in traveling waves that are time-periodic in a comoving frame. In this section, we therefore make precise this notion and show how to adapt the above dimension counting arguments. We refer to $[22,20,28,12,29,30]$ for background on this type of equation.

Consider a modulated traveling-wave solution $u=u(x-s t, \omega t), u(\xi, \tau)=u(\xi, \tau+2 \pi)$ to the reaction-diffusion system (4.1). This particular solution to a reaction-diffusion system solves a parabolic equation with time-periodic boundary conditions, which in many respects should be considered as a degenerate elliptic boundary-value problem; see for instance [29]. We nevertheless rewrite the equation for $u$ as a first-order system in the spatial variable $\xi$, setting $w=\left(u, u_{\xi}\right)(\xi, \tau)$, with $\tau=\omega t \in S^{1}=\mathbb{R} / 2 \pi \mathbb{Z}$, and obtain the modulated traveling-wave equation,

$$
w_{\xi}=A w+G(w)
$$

Here,

$$
A=\left(\begin{array}{cc}
0 & 1 \\
D^{-1} \omega \partial_{\tau} & -D^{-1} s
\end{array}\right), \quad G(w)=\left(\begin{array}{c}
0 \\
F(u)
\end{array}\right) .
$$

We regard this equation as a dynamical system on $H^{1}\left(S^{1}, \mathbb{R}^{N}\right) \times H^{1 / 2}\left(S^{1}, \mathbb{R}^{N}\right)$, with an underlying rotational symmetry acting through the temporal shift $w(\xi, \tau) \mapsto w(\xi, \tau+\varphi)$, $\varphi \in S^{1}$. Note that, within the fixed point space $\operatorname{Fix}\left(S^{1}\right)$ lie solutions that are independent of $\tau$, that is, pure traveling wave solutions $u=u(x-c t)$.

Spatially homogeneous equilibria of the reaction-diffusion system correspond to equilibria of this modulated traveling-wave equation contained in $\operatorname{Fix}\left(S^{1}\right)$. Spatially periodic, temporally steady solutions correspond to periodic orbits, or, more precisely, relative equilibria with respect to the $S^{1}$-symmetry: $w(\xi, \tau)=w_{*}(x)=w_{*}\left(\xi+\frac{s}{\omega} \tau\right)$. Note that necessarily $w_{*}(x)=$ $w_{*}(x+L)$ with wavelength $L=2 \pi / k, k=\frac{\omega}{s}$. Somewhat more generally, we could consider spatio-temporally periodic wave trains, $w_{*}\left(\xi-\frac{\tau}{k}\right)$ with period $\frac{2 \pi}{k}$ and speed $\frac{\omega}{k}$. If $k \neq-\frac{\omega}{s}$, those wave trains are moving in the steady frame with speed $\frac{\omega}{k}+s$. The modulated traveling waves that we are interested in connect the spatially homogeneous equilibrium $w_{+}=w(\tau) \equiv\left(u_{+}, 0\right)^{T}$ at $\xi=+\infty$ with a homogeneous equilibrium $w_{-}(\tau)=\left(u_{-}, 0\right)$, or with a pattern $w_{-}(\xi, \tau)=$ $w_{*}\left(\xi+\frac{s}{\omega} \tau\right)$. In other words, we are interested in intersections of the stable manifold of $w_{+}$and the unstable manifold of $w_{-}$. One can show that under (normal) hyperbolicity assumptions, 
these stable and unstable manifolds exist and are smooth, at least locally near a solution to the nonlinear problem [23, 29].

\subsection{Robustness — the role of Fredholm and Morse indices}

Our strategy for addressing the question of robustness of a given time-periodic invasion front is as follows. Suppose that we are given a fixed modulated traveling wave. Near such a front, we construct center-stable and center-unstable manifolds and ask if the intersection is transverse. Using smooth dependence of these manifolds on parameters, we can conclude robustness when the intersection is transverse, that is, the sum of tangent spaces spans the ambient phase space. To make this more precise, define the injection map near a modulated wave $w$,

$$
\iota: T_{w(0)} W_{+}^{c s} \times T_{w(0)} W_{-}^{c u} \mapsto Y, \quad\left(w^{\mathrm{cs}}, w^{\mathrm{cu}}\right) \mapsto w^{\mathrm{cs}}-w^{\mathrm{cu}} .
$$

Transversality is equivalent to surjectivity of $\iota$. Since modulated waves come in families, $\iota$ necessarily possesses a kernel. Clearly, $\xi$ - and $\tau$-derivatives contribute to the kernel.We define the linear multiplicity $i_{\mathrm{lm}}$ as the minimal dimension of the kernel. For generic travelingwave problems, this dimension is one, with kernel spanned by the derivative of the traveling wave. For generic modulated wave problems, this dimension is 2 , since the time-derivative contributes to the kernel, too. In our case of precipitation kinetics, it is 3: robust modulated waves come in families parameterized by the mass $m$, position $\xi$, and phase $\tau$; see Lemma 4.21 and Remark 4.22, below. The following definition formalizes this robustness concept.

Definition 4.4 We say that a modulated wave is robust by dimension counting if $\iota$ is Fredholm with index at least $i_{\mathrm{lm}}$. In other words, we require that $\iota$ may be onto after taking into account the necessary multiplicities of waves.

Similarly to the finite-dimensional case, one can determine the Fredholm index of $\iota$ from dimensions of stable and unstable manifolds, although these are both infinite-dimensional. One therefore defines relative Morse indices at the asymptotic state $w_{ \pm}$by comparing unstable eigenspaces to a fixed reference subspace. A convenient choice, which also normalizes the relative Morse index, is the unstable subspace of the system with $f^{\prime}=-\mathrm{id}$. One compares dimensions between subspaces in a straightforward fashion by computing the Fredholm index of the spectral projection, restricted to the reference subspaces; we refer to [30] for more details and alternative characterizations of relative Morse indices. All these (equivalent) definitions allow for the computation of relative Morse indices using crossing numbers as in Section 4.1.3.

Proposition 4.5 [28, 30] Suppose that the relative Morse indices of the asymptotic states are given by $i_{ \pm}$and that the total multiplicity of neutral modes is $n_{ \pm}$. Then the map $\iota$ is Fredholm with index $i(\iota)=i_{-}-i_{+}+n_{-}$. In particular, we have robustness by dimension counting if the multiplicity index $i_{\text {mult }}$ is non-negative,

$$
i_{\text {mult }}:=i_{-}+n_{-}-i_{+}-i_{\operatorname{lm}} \geq 0 .
$$

In the following, we compute relative Morse indices using the same homotopies as in the finitedimensional case, Section 4.1. Since crossing numbers are finite, one obtains relative Morse indices by simple bordering lemmas for Fredholm operators; see again [30]. 


\subsection{Computing relative Morse indices — homogeneous equilibria}

We consider spatially homogeneous equilibria, first. A short direct calculation shows that the linearization of the modulated traveling-wave ODE (4.3) possesses solutions of the form $w_{0} \mathrm{e}^{\nu \xi+\mathrm{i} \ell \tau}, w_{0} \in \mathbb{R}^{2 N}$, when $\nu, \ell$ solve

$$
d_{s}(\mathrm{i} \ell \omega, \nu)=d_{0}(\mathrm{i} \ell \omega-s \nu, \nu)=0
$$

where $d_{s}$ is the dispersion relation associated with the linearization at the homogeneous equilibrium, $d_{s}(\lambda, \nu)=\operatorname{det}\left(D \nu^{2}+s \nu+F^{\prime}-\lambda\right)$. In analogy to the definition in Section 4.1, we define resonant modes as follows.

Definition 4.6 (Resonant modes - modulated traveling waves) We say that the wavenumber $k$ corresponds to a resonant neutral mode $(\mathrm{i} \ell \omega, \mathrm{i} k), \ell \in \mathbb{Z}, k \in \mathbb{R}$, if $d_{s}(\mathrm{i} \ell \omega, \mathrm{i} k)=$ $d_{0}(\mathrm{i} \ell \omega-s \mathrm{i} k, \mathrm{i} k)=0$. Similarly, we define resonant growth modes $(\lambda+\mathrm{i} \ell \omega, \mathrm{i} k), \lambda>0$, $\ell \in \mathbb{Z}, k \in \mathbb{R}$, through the condition $d_{s}(\lambda+\mathrm{i} \ell \omega, \mathrm{i} k)=0$. In both cases, we call $k$ a resonant spatial wavenumber and $\ell$ the temporal harmonic.

We say that a resonant growth mode $(\lambda+\mathrm{i} \ell \omega, \mathrm{i} k)$ is temporally simple when $\partial_{\lambda} d_{s}(\lambda+\mathrm{i} \ell \omega, \mathrm{i} k) \neq$ 0 .

We define the spatial multiplicity of simple resonant modes $(\lambda+\mathrm{i} \ell \omega, \mathrm{i} k)$ as the sum of the orders of the root $\nu=\mathrm{i} k$ of $d_{s}(\lambda+\mathrm{i} \ell \omega, \cdot)$. The total spatial multiplicity of $\lambda$ is the sum over all multiplicities of resonant spatial wavenumbers and temporal harmonics associated with $\lambda$.

Note that resonance now refers to linear growth modes that oscillate with the same temporal period as the primary modulated traveling wave, or, in other words, they are (higher) harmonics to the traveling-wave frequency.

Using [30], one can now prove Lemma 4.2 for the modulated-wave equation. The proof is the same as in the case of the traveling-wave equation and will be omitted.

Lemma 4.7 Assume that neutral and growing modes are simple and have nonvanishing group velocity $s_{\mathrm{g}}$. Denote by $m_{ \pm}$the total multiplicities corresponding to positive and negative group velocities. Then the relative Morse index of $u$ in the traveling-wave equation is $m_{+}-m_{-}$.

\subsection{Computing relative Morse indices — periodic patterns}

One can treat the linearization at periodic patterns in a similar fashion. Consider the linearization at a time-periodic traveling wave $w_{*}\left(k_{*} \xi-\omega t\right), w_{*}(0)=w_{*}(2 \pi)$, with $k_{*} \neq 0$, and phase speed $s_{\mathrm{p}}=\frac{\omega}{k_{*}}$ in the frame moving with speed $s$. One can show [29] that solutions to the linearized equations can be written in the form $w_{0}\left(k_{*} \xi-\omega t\right) \mathrm{e}^{\lambda t+\nu \xi}$, with a $2 \pi$-periodic function $w_{0}$. Substituting this ansatz into the parabolic equation, one obtains a linear elliptic boundary-value problem for $w_{0}=\left(u_{0}, u_{0, \xi}\right)$,

$\left[D\left(k_{*} \partial_{y}+\nu\right)^{2}+s\left(k_{*} \partial_{y}+\nu\right)+F^{\prime}\left(u_{*}(y)\right)+\omega \partial_{y}-\lambda\right] u_{0}=0, \quad u_{0}(0)=u_{0}(2 \pi), u_{0}^{\prime}(0)=u_{0}^{\prime}(2 \pi)$, 
or,

$$
\left[D\left(k_{*} \partial_{y}+\nu\right)^{2}+\tilde{s}\left(k_{*} \partial_{y}+\nu\right)+F^{\prime}\left(u_{*}(y)\right)-\tilde{\lambda}\right] u_{0}=0, \quad u_{0}(0)=u_{0}(2 \pi), u_{0}^{\prime}(0)=u_{0}^{\prime}(2 \pi),
$$

where $\tilde{s}=s+s_{\mathrm{p}}=\frac{\tilde{\omega}}{k_{*}}$ and $\tilde{\omega}=k_{*} \tilde{s}$ are the speed and frequency of the wave train in the steady frame, and $\tilde{\lambda}=\lambda+\frac{\omega}{k_{*}} \nu$ is the shifted eigenvalue. Of course, $\tilde{\omega}=0$ for standing patterns $u_{*}(x)$. Solutions to this boundary-value problem exist if the period-map to the associated first-order ODE possesses a non-trivial fixed point. Therefore, consider the flow to the non-autonomous ODE

$$
\begin{aligned}
& k_{*} u_{y}=v \\
& k_{*} v_{y}=-D^{-1}\left(\tilde{s} v+F^{\prime}\left(u_{*}(y)\right)-\tilde{\lambda}\right),
\end{aligned}
$$

with associated period map $\Phi_{\tilde{\lambda}}(u(0), v(0))=(u(2 \pi), v(2 \pi))$. Solutions to the boundary-value problem (4.5) correspond to Floquet exponents of $\Phi_{\tilde{\lambda}}$, that is, to roots of

$$
d_{s}(\lambda, \nu):=d_{0}\left(\lambda-s_{\mathrm{p}} \nu, \nu\right), \quad d_{0}(\tilde{\lambda}, \nu)=\operatorname{det}\left(\Phi_{\tilde{\lambda}}-\mathrm{e}^{2 \pi \nu / k_{*}}\right) .
$$

Note that we need to restrict to $\operatorname{Im} \nu \in\left[0, \mathrm{i} k_{*}\right)$, thus eliminating the Floquet symmetry, when we count multiplicities of roots.

With this definition of the dispersion relation $d_{s}(\lambda, \nu)$, one can define resonant modes as in Definition 4.6, while restricting the allowed set of $\nu$ to $0 \leq \operatorname{Im} \nu<\mathrm{i} k_{*}$, and prove Lemma 4.7.

We also consider the special case where the wave train is stationary in a steady frame, $u_{*}(\xi-$ $s t)=u_{*}(x)=u_{*}(-x), s_{\mathrm{p}}=-s, \tilde{s}=0$. In this case, the linearized ODE (4.6) is reversible with respect to the reverser $R(u, v)=(u,-v)$ in the following sense. We have that $\Phi_{\lambda}=R \Phi_{\lambda}^{-1} R$, so that $\operatorname{det} \Phi_{\lambda}=1$ and

$$
\operatorname{det}\left(\Phi_{\lambda}-\rho\right)=\operatorname{det}\left(\rho \Phi_{\lambda}\right) \operatorname{det}\left(\Phi_{\lambda}^{-1}-\rho^{-1}\right)=\rho^{2 N} \operatorname{det}\left(\Phi_{\lambda}-\rho^{-1}\right) .
$$

Thereby, the function $\tilde{d}(\lambda, \rho):=\rho^{-N} \operatorname{det}\left(\Phi_{\lambda}-\rho\right)$ satisfies $\tilde{d}(\lambda, \rho)=\tilde{d}\left(\lambda, \rho^{-1}\right)$.

In summary, the modified dispersion relation

$$
\tilde{d}_{0}(\lambda, \nu):=\mathrm{e}^{-N \pi \nu / k_{*}} d_{0}(\lambda, \nu)
$$

satisfies $\tilde{d}_{0}(\lambda, \nu)=\tilde{d}_{0}(\lambda,-\nu)$, can be written as a real analytic function $\hat{d}\left(\lambda, \nu^{2}\right)$, and possesses the same roots as $d_{0}$. For $\lambda$ real and $\nu$ purely imaginary, $\partial_{\lambda} \tilde{d}_{0}$ is therefore real, and $\partial_{\nu} \tilde{d}_{0}$ purely imaginary. This proves the following lemma.

Lemma 4.8 Suppose that $u_{*}(x)=u_{*}(-x)$ is a spatially periodic, even function. Then group velocities for real eigenvalues $\lambda \in \mathbb{R}$ and purely imaginary $\nu$ vanish in the steady frame. In particular, the relative Morse index is given by $-m_{-}$, the number of resonant neutral and growing modes, provided that the spectrum is real and simple, that is, when $d_{0}(\lambda, \mathrm{i} k) \neq 0$ for $\lambda \notin \mathbb{R}$, and $\partial_{\lambda} d_{0}(\lambda, \mathrm{i} k) \neq 0$ whenever $d_{0}(\lambda, \mathrm{i} k)=0$. 


\subsection{Pulled fronts with critical decay - Morse indices and the absolute spectrum}

The linear analysis near the unstable state predicts spatial decay of fronts with asymptotics $\xi \mathrm{e}^{\nu_{\text {lin }} \xi}$ for $\xi \rightarrow \infty$. Our computations of Morse indices allow for fronts with decay slower than this rate. The following definition restricts the class of fronts to fronts with this critical linear decay rate.

Definition 4.9 (Pulled front with critical decay) We say that $u(x-s t, \omega t)$ is a pulled front with critical decay if $u(\xi, \tau)=u(\xi, \tau+2 \pi), s=s_{\text {lin }}, \omega=\omega_{\text {lin }}$, and if $u$ possesses critical asymptotics $\xi \mathrm{e}^{\nu_{\operatorname{lin}} \xi}$ : we require that there exists $u_{\infty} \in H^{1}\left(S^{1}, \mathbb{R}^{N}\right)$ and $\delta>0$ such that in $H^{1}\left(S^{1}, \mathbb{R}^{N}\right)$,

$$
\mathrm{e}^{\delta \xi}\left(u(\xi, \cdot)-\xi \mathrm{e}^{\nu_{\operatorname{lin}} \xi} u_{\infty}(\cdot)\right) \rightarrow 0
$$

In the following, we show that one can associate a relative Morse index to the type of decay prescribed in Definition 4.9. Recall the construction of the dispersion relation $d_{s}$ in (4.4).

Lemma 4.10 Suppose $\nu_{\text {lin }}$ and $\bar{\nu}_{\text {lin }}$ are double roots of the dispersion relation $d_{s}(\mathrm{i} \ell \omega, \nu)$ for $\ell= \pm 1, s=s_{\operatorname{lin}}, \omega=\omega_{\text {lin }}$. Moreover, assume that there are no other roots with $\operatorname{Re} \nu=$ $\operatorname{Re} \nu_{\text {lin }}$ for any $|\ell| \neq 1$. Then there exists a smooth strong stable manifold of solutions with critical asymptotic decay. Finally, let $i_{\mathrm{s}}$ be the number of roots (counted with multiplicity) in $\left\{\operatorname{Re} \nu_{\text {lin }}<\operatorname{Re} \nu \leq 0\right\}$. Then the relative Morse index of the tangent space of this strong stable manifold is $i_{+}+i_{\mathrm{s}}$.

Proof. The proof is a consequence of the existence of strong stable manifolds $[23,30,29]$, and the straightforward dimension counting.

Definition 4.11 (Morse index of critical decay) We refer to the relative Morse index of the strong stable manifold as $i_{\mathrm{ss}}=i_{+}+i_{\mathrm{s}}$.

The assumptions in Lemma 4.10 are typical in the sense that one does not assume the linear spreading speed to be caused by more modes with critical decay than necessary. On the other hand, the lemma shows that the dimension counting for pulled fronts with critical decay may be different than the one for pulled fronts in general, due to the presence of spatial eigenvalues with weaker decay than $\operatorname{Re} \nu_{\text {lin }}$.

In order to calculate $i_{\mathrm{ss}}$ under typical conditions, we need to recall the characterization of the absolute spectrum, given in Section 2.2. We defined the absolute spectrum $\Sigma_{\text {abs }}$ as the set of $\lambda$ such that the roots of $d_{s}(\lambda, \nu)$ satisfy $\operatorname{Re} \nu_{N}=\operatorname{Re} \nu_{N+1}$ when all $2 N$ roots are ordered by real part $\operatorname{Re} \nu_{j} \leq \operatorname{Re} \nu_{j+1}$. The absolute spreading speed $s_{\text {abs }}$ was then defined as the largest $s$ so that the absolute spectrum intersects the closed right half plane. Of course, for $s=s_{\text {abs }}$, there exists a finite collection $\Sigma_{\mathrm{abs}} \cap \mathrm{i} \mathbb{R}$ of values of $\lambda \in \mathrm{i} \mathbb{R}$ in the absolute spectrum. If $\nu_{N}=\nu_{N+1}$ at such a $\lambda$, then it corresponds to a double root with pinching condition. We refer to the subset $\Sigma_{\text {abs }} \cap\{\operatorname{Re} \lambda \geq 0\}$ where $\nu_{N} \neq \nu_{N+1}$ as remnant modes; see [27, 26]. 
Definition 4.12 (Simple spreading via pointwise growth) We say spreading is caused by simple pointwise growth if for $s=s_{\text {abs }}$

(i) $\Sigma_{\mathrm{abs}} \cap \mathrm{i} \mathbb{R}$ consists of $\lambda=0$ or of $\lambda= \pm \mathrm{i} \omega$. In both cases, we require that $\lambda$ is a simple double root for a unique value $\nu$ : $\partial_{\nu} d=0, \partial_{\lambda} d \neq 0, \partial_{\nu \nu} d \neq 0$.

(ii) There are no remnant modes.

In particular, $s_{\mathrm{abs}}=s_{\text {lin }}$.

Remark 4.13 We suspect that simple spreading via pointwise growth holds for almost all values of $\gamma$ and $a$ in our example. In particular, we suspect that there are no remnant instabilities, that is, (ii) holds for all parameter values. Condition (i) is a generic condition and should be satisfied in most examples. The calculations in Section 2 show that simple spreading via pointwise growth holds in the weak-coupling and in the Cahn-Hilliard regime.

Lemma 4.14 Assume simple spreading via pointwise growth. Then $i_{\mathrm{ss}}=-2\left(\omega_{\mathrm{lin}} \neq 0\right)$ or $i_{\mathrm{ss}}=-1\left(\omega_{\mathrm{lin}}=0\right)$.

Proof. A homotopy from $\lambda=+\infty$ shows that we can always separate by real part roots coming from $\nu=+\infty$ and roots coming from $\nu=-\infty$, until $\lambda \in \mathrm{i} \mathbb{R}$, since otherwise we would cross a curve of absolute spectrum. At $\lambda \in \Sigma_{\text {abs }} \cap \mathbb{i} \mathbb{R}$, precisely two of those roots meet at $\nu_{\text {lin }}$, therefore decreasing the relative number of roots with $\operatorname{Re} \nu>\operatorname{Re} \nu_{\text {lin }}$ by one. It remains to show that we have $\operatorname{Re} \nu_{\text {lin }} \leq 0$. This can be readily established by following the double root $\lambda_{\mathrm{dr}}$ of the dispersion relation in $s$. Differentiating the dispersion relation, one readily finds that $\partial_{s} \lambda_{\mathrm{dr}}=\nu$. Since $s$ was maximal with the property that $\operatorname{Re} \lambda_{\mathrm{dr}}>0$, we can conclude that $\partial_{s} \lambda_{\mathrm{dr}} \leq 0$ and therefore $\operatorname{Re} \nu_{\text {lin }} \leq 0$. This proves the lemma.

Corollary 4.15 (Robustness of invasion fronts with critical decay) Assume simple spreading via pointwise growth in a generic reaction-diffusion system, that is, $i_{\mathrm{lm}}=1$ for steady $\left(\omega_{\mathrm{lin}}=0\right)$ and $i_{\mathrm{lm}}=2$ for oscillatory $\left(\omega_{\mathrm{lin}}=0\right)$ propagation. Then pulled fronts with critical decay are robust by dimension counting if the pattern in the wake does not possess resonant unstable or neutral modes except for possibly a single neutral mode associated with the translation of the pattern. .

This concept can be further generalized including possible resonant unstable modes such that the multiplicity of modes with $c_{\mathrm{g}}$ exceeds the multiplicity of modes with $c c_{\mathrm{g}}<0$.

One can also generalize to harmonic invasion front, where the linear frequency $\omega_{\text {lin }}$ is an integer multiple of the frequency of the invasion front. Resonant unstable modes are then defined relative to this frequency of the invasion front.

\subsection{Counting pushed fronts}

The counting arguments given above can be easily adapted to the study of pushed fronts as defined in Section 3.1. According to the definition, there, pushed fronts lie in the manifold 
associated with the spatial eigenvalues $\nu_{1}, \ldots, \nu_{N}$. When $\nu_{N}<0$, this manifold corresponds to a strong stable manifold with Morse index 0. As a consequence, the linearization at pushed fronts that leave behind a state without neutral or growing resonant modes is Fredholm with index 0 . The one-dimensional kernel generated by translations of the front profile is compensated for by the parameter $s$. In other words, pushed fronts are robust by dimension counting. Similarly, one can show that modulated pushed fronts are robust by dimension counting. Again, the relative Morse index of the strong stable subspace is 0 , so that the linearization is Fredholm with index 0 . The two-dimensional kernel spanned by translation and time-shift is compensated for by the free parameters $s$ and $\omega$.

All these counting arguments fail when $R e \nu_{N}>0$. For simple spreading via pointwise growth, we have $\operatorname{Re} \nu_{\text {lin }}<0$. Since the branch of absolute spectrum that ends in the associated double root points into the stable half plane, $\operatorname{Re} \nu_{N}<\operatorname{Re} \nu_{\text {lin }}<\operatorname{Re} \nu_{N+1}$ for $s \gtrsim s_{\text {lin }}$. Even in the simple example with variational structure that we have studied here, it appears difficult to prove that $\operatorname{Re} \nu_{N}<0$ for all $s>s_{\text {lin }}$.

In a similar vein, it is difficult to show that $\operatorname{Re} \nu_{N}<\operatorname{Re} \nu_{\text {lin }}$, so that pushed fronts are actually steeper than the linear prediction. In simple cases, one can verify this property explicitly [35], but general criteria do not appear to be available.

\subsection{The case of precipitation kinetics}

We apply the results from the previous sections to the case of precipitation kinetics. We set $u=(c, e)^{T}, D=\operatorname{diag}(1, \kappa)$, and $F=f(c, e)(-1,1)^{T}$. We find the modulated traveling-wave equation,

$$
w_{\xi}=A w+F(w)
$$

Here,

$$
A=\left(\begin{array}{cccc}
0 & 0 & 1 & 0 \\
0 & 0 & 0 & 1 \\
\omega \partial_{\tau} & 0 & -s & 0 \\
0 & \kappa^{-1} \omega \partial_{\tau} & 0 & -\kappa^{-1} s
\end{array}\right), \quad F(w)=\left(\begin{array}{c}
0 \\
0 \\
f(c, e) \\
-\kappa^{-1} f(c, e)
\end{array}\right)
$$

We are interested in heteroclinic connections from the (temporally) unstable equilibrium $w_{+}=\left(c_{+}, e_{+}, 0,0\right)$ with $c_{+}=0, e_{+}=a$ to a spatially homogeneous or periodic state $w_{-}=\left(c_{-}, e_{-}, c_{-, \xi}, e_{-, \xi}\right)$.

We shall first exploit the gradient structure (1.4)-(1.5) in order to show that there are no complex temporal eigenvalues $\lambda$ to the linearization at a spatially periodic equilibrium $\left(c_{*}, e_{*}\right)(x)$.

Lemma 4.16 Assume $\gamma>0$ and $0<\kappa<1$. Let $d_{0}(\lambda, \nu)$ be the dispersion relation for the linearization as defined in (4.4) for spatially homogeneous and (4.7) for spatially periodic equilibria. Then $d_{0}(\lambda, \mathrm{i} k) \neq 0$ when $\lambda \notin \mathbb{R}$.

Proof. We consider the case of a spatially periodic equilibrium of minimal period $L$, only, which comprises the case of spatially homogeneous equilibria.

First, note that $d_{0}\left(\lambda_{0}, \mathrm{i} k_{0}\right)=0$ for some $\lambda_{0}$ with $\operatorname{Im} \lambda_{0} \neq 0$ implies that we can find $\lambda_{1}$ close to $\lambda_{0}$ with $\operatorname{Im} \lambda_{1} \neq 0$ and $k_{1}=(2 \pi / L)(p / q), p / q \in \mathbb{Q}$ such that $d\left(\lambda_{1}, \mathrm{i} k_{1}\right) \neq 0$, by analyticity of $d_{0}$ 
and Rouché's theorem. In particular, this implies that we would find a complex eigenvalue of the linearization considered on a finite domain $x \in[0, q L]$ with periodic boundary conditions. Next, notice that the linearization can be written in the form $\mathcal{L}=-M \mathcal{A}$, where $M$ was defined in (1.5) and $\mathcal{A}$ is the second derivative of the energy $W$, defined in (1.4). One easily verifies that $\mathcal{L}$ possesses a codimension-one invariant subspace $X_{0}$ of $L^{2}\left([0, q L], \mathbb{R}^{2}\right)$, defined by $\int(c+e)=0$, that is, $\mathcal{L}$ is densely defined on $X_{0}$ and maps into $X_{0}$. Moreover, $\mathcal{L}$ possesses compact resolvent and pure point spectrum as an elliptic operator on a bounded domain. We claim that $\mathcal{L}$, restricted to this subspace does not possess complex eigenvalues.

We endow $X_{0}$ with the (formal) scalar product $\langle u, v\rangle:=\left(M^{-1} u, v\right)_{L^{2}}$. One easily verifies that this scalar product is well defined on $X_{0}$, and that

$$
\langle\mathcal{L} u, v\rangle=-(\mathcal{A} u, v)_{L^{2}}=-(u, \mathcal{A} v)_{L^{2}}=\langle u, \mathcal{L} v\rangle,
$$

whenever $u, v$ belong to the domain of $\mathcal{L}$ in $X_{0}$. Complexifying the scalar product, one now readily precludes complex eigenvalues in $X_{0}$ and then in $L^{2}$.

Lemma 4.17 The relative Morse index $i$ of a spinodal unstable homogeneous state is

$$
i=-1-2\left[k_{\max } / k_{\text {lin }}\right] \geq 3 .
$$

The relative Morse index for a homogeneous unstable homogeneous state is

$$
i=-2-2\left[k_{\max } / k_{\text {lin }}\right] \geq 4 .
$$

Here, $[a]=\max \{j \in \mathbb{Z}, j \leq a\}, k_{\max }$ is the maximal unstable wavenumber, and $k_{\operatorname{lin}}=\omega_{\operatorname{lin}} / s_{\operatorname{lin}}$ is the wavenumber selected by the instability; see Section 2.6 for definitions. Spinodal refers to region (ii) in Figure 1.1, homogeneous to regions (iii) and (iv).

Proof. Resonant modes satisfy $d(\lambda+\mathrm{i} \omega \ell-s \mathrm{i} k, \mathrm{i} k)=0$ for some real $\lambda \geq 0$ and $\ell \in \mathbb{Z}$. By Lemma $4.16, k=\ell \omega / s=\ell k_{\text {sel }}$. Nonnegative modes $\lambda(k) \geq 0$ exist for $k=0$ (neutral) and $|k| \leq k_{\text {lim }}$. All modes are simple. Resonant neutral or growth modes therefore exist for any $|\ell| \leq k_{\text {lim }} / k_{\text {sel }}$. The count now follows from Lemma 4.8 , provided that we can show that $k_{\lim } \geq k_{\text {sel }}$. For this, note that $\lambda=\mathrm{i} \omega$ yields a double root of $d_{s}$ with pinching condition. In particular, there is a root $\nu$ of $d(\lambda-s \nu, \nu)$ that crosses the imaginary axis as $\operatorname{Re} \lambda=\mathrm{i} \omega+\eta$, $\eta \rightarrow+\infty$. This gives the desired root $d\left(\mathrm{i} \omega+\eta_{0}-s \nu_{0}, \nu_{0}\right)$ with $\nu_{0} \in \mathrm{i} \mathbb{R}$.

Of course, the lemma also gives information on the Morse index of spatially homogeneous equilibria $w_{-}$in the wake of invasion fronts.

From the plot of $k_{\max }$ and $k_{\text {lin }}$ in Figure 2.1, one sees that the maximal allowed wavenumber is larger than wavenumbers selected by the invasion process and wavenumbers with maximal temporal growth. In the Cahn-Hilliard limit $\gamma \rightarrow 1 /(\kappa+1)$, one can check that $1<k_{\max } / k_{\text {sel }}<$ 2. The second resonant mode becomes relevant, and thus the Morse index changes around $\gamma=0.7$ for $\kappa=0.1$.

We next investigate the relative Morse index of spatially periodic patterns. Let $(e, c)(x)$ be a periodic pattern with minimal period $L$, wavenumber $k_{*}=2 \pi / L$, so that $\omega_{\operatorname{lin}}=s_{\operatorname{lin}} k_{*}$. In 
other words, we consider periodic patterns with the selected wavenumber, excluding harmonics $k_{*}=j k_{\text {lin }}$, for now. Moreover, we say that the periodic pattern is linearly stable if there are no positive eigenvalues to the linearization when considered on functions with period $L$, and if the linearization possesses a double eigenvalue $\lambda=0$. We borrow the following lemma from $[25]$; see also $[18,11]$ for more comprehensive (but slightly different) stability information.

Lemma 4.18 [25] Given a periodic pattern with minimal period L, there is a family of patterns parameterized by $\mu=c+\kappa e$, of period $L$, and mass $m(\mu)$. We have the following information on stability.

(i) All periodic patterns are unstable with respect to essential spectrum when considered on $x \in \mathbb{R}$.

(ii) Periodic patterns possess a double zero eigenvalue when considered with periodic boundary conditions, provided $m^{\prime}(\mu) \neq 0$. The zero eigenvalue is triple if $m^{\prime}(\mu)=0$.

(iii) Periodic patterns possess Morse index $i=2 j-1$ for $m^{\prime}(\mu)>0$ and $i=2 j$ for $m^{\prime}(\mu) \leq 0$ when considered with $j L$-periodic boundary conditions.

Lemma 4.19 Given a linear spreading speed $s_{\operatorname{lin}}$ and frequency $\omega_{\mathrm{lin}}$, consider a spatially periodic pattern with minimal period $L=2 \pi / k_{\operatorname{lin}}, k_{\operatorname{lin}}=\omega_{\operatorname{lin}} / s_{\operatorname{lin}}$, and with $m^{\prime}(\mu) \neq 0$. Then the relative Morse index of this pattern in the modulated traveling-wave equation is -2 when $m^{\prime}(\mu)<0$, and -3 when $m^{\prime}(\mu)>0$. The zero-eigenvalue of the pattern is geometrically double in both cases.

Proof. The proof is similar to the proof of Lemma 4.17. Since the spectrum is real in a steady frame, we have that $k=\ell \omega / s=\ell k_{\text {sel }}$. Since we assumed that the period of the periodic pattern equals the selected wavenumber, and $k \in[0,2 \pi / L)$ by its definition as a spatial Floquet exponent, we conclude that $k=0$ and resonant growth modes are necessarily of the same period as the periodic pattern. Counting unstable eigenvalues and using Lemmas 4.8 and 4.18,we immediately obtain the formulas claimed in the lemma, with the caveat that Lemma 4.8 only applies to simple resonant modes. It therefore remains to show that both eigenvalues at 0 actually decrease the relative Morse index. We therefore expand the dispersion relation $d(\lambda, \nu)=d_{11} \lambda^{2}+d_{22} \nu^{2}+\mathrm{O}\left(|\nu|^{4}+\left|\nu^{2} \lambda\right|+|\lambda|^{3}\right)$. We claim that $d_{22}=0$. This would give that $d_{s}(\lambda, \nu)=d_{11}(\lambda-s \nu)^{2}+\mathrm{O}\left((|\lambda|+|\nu|)^{3}\right)$, and therefore $\lambda=s \nu+\mathrm{O}\left(\nu^{2}\right)$, the desired result. In order to see that $d_{22}=0$, notice that at $\lambda=0$, the multiplicity of the root $\nu$ counts the multiplicity of the zero Floquet exponent of the periodic pattern in the steady-state ODE. This zero Floquet exponent is geometrically double since translation and derivative with respect to the conserved quantity provide eigenvectors. Reversibility gives at least two generalized eigenvectors, so that the multiplicity is at least four. This proves the lemma.

Remark 4.20 A few remarks on the assumptions in the lemma are in order.

(i) Stable periodic patterns exist in many cases. One can see this for instance by minimizing the energy (1.4) on a subspace with fixed mass of periodic functions. When spatially 
homogeneous equilibria with the given mass are all unstable, the minimizer is a spatially periodic pattern. In particular, stable periodic patterns always exist when $\gamma>1 / 4$.

(ii) On the other hand, there do not exist any stable periodic patterns of bounded period for small $\gamma$. This can be readily seen by perturbing from the $\gamma=0$ limit, where $m^{\prime}(\mu)=1>$ 0: all periodic patterns in the Allen-Cahn equation are unstable.

Lemma 4.21 The (modulated) traveling-wave equation conserves mass. More precisely, for any solution $w(\xi)$, the pointwise time averages $\int_{\tau}(c+e)(\xi, \tau) \mathrm{d} \tau \equiv m$ are equal in the two limits $\xi \rightarrow \pm \infty$.

Proof. Solutions are smooth by parabolic interior regularity, so that we have the pointwise equality

$$
(c+\kappa e)_{\xi \xi}+s(c+e)_{\xi}=\omega \partial_{\tau}(c+e) .
$$

Integrating over $\tau$ and using periodicity in $\tau$, this gives

$$
\int_{\tau}\left((c+\kappa e)_{\xi \xi}+s(c+e)_{\xi}\right) \mathrm{d} \tau=0,
$$

and, upon integration in $\xi$,

$$
\int_{0}^{2 \pi}\left((c+\kappa e)_{\xi}+s(c+e)\right) \mathrm{d} \tau=: s m_{*},
$$

a constant independent of $\xi$. For equilibria $(c, e)(x)$, we have $c+\kappa e \equiv \mu$, spatially constant, so that

$$
\int_{\tau}(c+e)(\xi, \tau) \mathrm{d} \tau \rightarrow m_{*}, \text { for } \xi \rightarrow \pm \infty
$$

Remark 4.22 In the following, we could pursue an alternative strategy and exploit the fact that $I[w(\xi, \cdot)]:=\int\left((c+\kappa e)_{\xi}+s(c+e)\right) \mathrm{d} \tau$ defines a first integral for the dynamics of the modulated traveling-wave equation. We could then restrict to the affine codimension-one subspaces of constant I, where neutral directions, but also ambient space dimension are reduced by one. We prefer to count indices in full space, and accounting for the neutral mode and additional multiplicities, caused by the conserved quantity, separately.

We are now ready to state robustness results for coherent pulled fronts, with and without critical decay. First, recall that spatially homogeneous equilibria come in one-parameter families, typically parameterized by the mass $m$. Therefore the number of neutral modes of $w_{+}$is one. Similarly, we already noticed that the number of neutral modes of spatially periodic equilibria is two as long as $m^{\prime}(\mu) \neq 0$.

In the terminology of Proposition 4.5, we have $n_{+}=1$ and $n_{-}=1$ for homogeneous equilibria and $n_{-}=2$ for spatially periodic equilibria with $m^{\prime}(\mu) \neq 0$. Moreover, we have $i_{\mathrm{lm}}=3$.

We summarize the results on relative Morse indices for asymptotic states $w_{ \pm}$in the following Tables 4.1 and 4.2. The wavenumbers $k_{\max }^{ \pm}$refer to maximal unstable wavenumber at the 


\begin{tabular}{llcll}
\hline pattern & stability & $i_{-}$ & $n_{-}$ & Reference \\
\hline spatially homogeneous & stable & -1 & 1 & Lemma 4.17 \\
\hline spatially homogeneous & spinodal unstable & $-1-2\left[k_{\max }^{-} / k_{\text {lin }}^{+}\right]$ & 1 & Lemma 4.17 \\
\hline spatially homogeneous & homogeneous unstable & $-2-2\left[k_{\max }^{-} / k_{\text {lin }}^{+}\right]$ & 1 & Lemma 4.17 \\
\hline spatially periodic & stable on $\mathbb{R} / L \mathbb{Z}$ & -2 & 2 & Lemma 4.19 \\
\hline spatially periodic & unstable on $\mathbb{R} / L \mathbb{Z}$ & -3 & 2 & Lemma 4.19 \\
\hline
\end{tabular}

Table 4.1: Relative Morse indices and number of neutral modes for patterns in the wake of invasion fronts.

\begin{tabular}{llcl}
\hline pattern & stability & $i_{+}$ & Reference \\
\hline spatially homogeneous & stable & -1 & Lemma 4.17 \\
\hline spatially homogeneous & spinodal unstable & $-1-2\left[k_{\max }^{+} / k_{\text {lin }}^{+}\right]$ & Lemma 4.17 \\
\hline spatially homogeneous & homogeneous unstable & $-2-2\left[k_{\max }^{+} / k_{\text {lin }}^{+}\right]$ & Lemma 4.17 \\
\hline
\end{tabular}

Table 4.2: Relative Morse indices and number for spatially homogeneous state ahead of invasion fronts.

asymptotic states $u_{ \pm}$at $\pm \infty$, defined in (1.8). The wavenumber $k_{\operatorname{lin}}^{+}$is the selected wavenumber at $u_{+}$.

The relative Morse indices for critical decay are given in Lemma 4.10. However, we need to correct for the neutral mode associated with variations in mass: we can consider the family of strong stable manifolds parameterized by varying mass and thereby augment the effective dimension $i_{\mathrm{ss}}$ by one without giving up on critical decay. This yields relative Morse indices as summarized in Table 4.3. From the relative Morse indices in Tables 4.1-4.3, one can readily

\begin{tabular}{llll}
\hline pattern & stability & $i_{\mathrm{ss}}$ & Reference \\
\hline spatially homogeneous & spinodal unstable & -3 & Lemma 4.10 \\
\hline spatially homogeneous & homogeneous unstable & -3 & Lemma 4.10 \\
\hline
\end{tabular}

Table 4.3: Relative Morse indices for coherent pulled fronts with critical decay.

conclude robustness by dimension counting as follows. Coherent pulled fronts are robust by dimension counting if

$$
i_{\text {mult }}=i_{-}+n_{-}-i_{+}-i_{\mathrm{lm}} \geq 0, \quad \text { for coherent pulled fronts, }
$$

and

$$
i_{\text {mult }}=i_{-}+n_{-}-i_{\mathrm{ss}}-i_{\mathrm{lm}} \geq 0, \quad \text { for fronts with critical decay; }
$$

see Proposition 4.5.

The following proposition condenses our counting arguments in simple predictions for occurrence of coherent pulled fronts and coherent pulled fronts with critical decay. 
Proposition 4.23 The following Tables 4.4 and 4.5 give multiplicity indices for coherent pulled fronts with and without critical decay. In the case of critical decay, we assumed simple spreading via pointwise growth, Definition 4.12. Fronts are robust by dimension counting if the table entry $i_{\text {mult }}$ is nonnegative. In particular, fronts leaving behind a stable or spinodal unstable pattern are robust by dimension counting. Fronts with critical decay leaving behind an unstable pattern are not robust by dimension counting. Fronts invading a homogeneous unstable state and leaving behind an unstable periodic pattern are robust by dimension counting but not when critical decay is imposed.

\begin{tabular}{lcccc}
\hline & homogeneous & homogeneous & perodic & periodic \\
stable & spinodal & stable & unstable \\
\hline $\begin{array}{l}\text { spinodal } \\
\text { unstable }\end{array}$ & $2\left[\frac{k_{\max }^{+}}{k_{\operatorname{lin}}^{+}}\right]-2 \geq 0$ & $2\left[\frac{k_{\max }^{+}}{k_{\operatorname{lin}}^{+}}\right]-2\left[\frac{k_{\max }^{-}}{k_{\operatorname{lin}}^{+}}\right]-2$ & $2\left[\frac{k_{\max }^{-}}{k_{\operatorname{lin}}^{+}}\right]-2 \geq 0$ & $2\left[\frac{k_{\max }^{-}}{k_{\operatorname{lin}}^{+}}\right]-3 \geq-1$ \\
\hline $\begin{array}{l}\text { homog. } \\
\text { unstable }\end{array}$ & $2\left[\frac{k_{\max }^{+}}{k_{\operatorname{lin}}^{+}}\right]-1 \geq 1$ & $2\left[\frac{k_{\max }^{+}}{k_{\operatorname{lin}}^{+}}\right]-2\left[\frac{k_{\max }^{-}}{k_{\operatorname{lin}}^{+}}\right]-1$ & $2\left[\frac{k_{\max }^{-}}{k_{\operatorname{lin}}^{+}}\right]-1 \geq 1$ & $2\left[\frac{k_{\max }^{-}}{k_{\operatorname{lin}}^{+}}\right]-2 \geq 0$ \\
\hline
\end{tabular}

Table 4.4: Multiplicity index $i_{\text {mult }}$ for coherent pulled fronts connecting a leading edge state (left column) to a pattern in the wake (top row).

\begin{tabular}{lcccc}
\hline & $\begin{array}{c}\text { homogeneous } \\
\text { stable }\end{array}$ & $\begin{array}{c}\text { homogeneous } \\
\text { spinodal }\end{array}$ & $\begin{array}{c}\text { perodic } \\
\text { stable }\end{array}$ & $\begin{array}{c}\text { periodic } \\
\text { unstable }\end{array}$ \\
\hline $\begin{array}{l}\text { spinodal } \\
\text { unstable }\end{array}$ & 0 & $-2\left[\frac{k_{\max }^{-}}{k_{\text {lin }}^{+}}\right] \leq 0$ & 0 & -1 \\
\hline $\begin{array}{l}\text { homog. } \\
\text { unstable }\end{array}$ & 0 & $-2\left[\frac{k_{\max }^{-}}{k_{\text {lin }}^{+}}\right] \leq 0$ & 0 & -1 \\
\hline
\end{tabular}

Table 4.5: Multiplicity index $i_{\text {mult }}$ for coherent pulled fronts with critical decay connecting a leading edge state (left column) to a pattern in the wake (top row).

\section{Proof.}

The table entries are obtained from Tables 4.1-4.3 and the formulas in (4.9) and (4.10). For instance, invasion of an unstable homogeneous state by an unstable periodic pattern gives $i_{-} \leq-2, n_{-}=1, i_{\mathrm{ss}}=-3, i_{\mathrm{lm}}=3$, so that $i_{\text {muult }} \leq-1$. On the other hand, $i_{+} \leq-4$, so that $i_{\text {mult }} \leq 1$ for fronts without restriction on critical decay.

\section{$5 \quad$ Numerical studies and discussion of results}

We illustrate our predictions with numerical simulations and point to a variety of other interesting phenomena.

We simulated (1.3) using finite differences and a semi-explicit scheme on $x \in[0, L]$ with Neumann boundary conditions. Initial conditions were taken to be $(c, e) \equiv(0, a)$ for $x \in$ 
$[0.08 \cdot L, L]$ and $(c, e) \equiv(a-1,1)$ for $x \in[0,0.08 \cdot L]$. All plots show $e(t, x)$ as a gray-scale plot with lighter color corresponding to larger values of $e$.

\subsection{Weak coupling}

Pushed-pulled transitions. For small $\gamma$, we predict a transition from pushed to pulled front propagation near $a=\kappa /(2-\kappa)$, when $\kappa<1 / 2$, or near $a=1 / 3$, when $\kappa>1 / 2$. In the first case, we expect the transition to be oscillatory due to the splitting of the double (in $\lambda$ ) double (in $\nu$ ) root into a pair (in $\lambda$ ) of complex double (in $\nu$ ) roots. In the latter case, we expect the transition to be stationary since a pair of real double roots crosses the imaginary axis.

Figure 5.1 shows the transition in the case $\kappa=0.1<0.5, \gamma=0.001$, with a predicted transition at $a_{*}(\gamma)=0.052+\mathrm{o}_{\gamma}(1)$. The transition actually occurs approximately at $a_{*}=$ 0.042. For $\gamma=10^{-4}$, the transition occurs at $a_{*} \sim 0.048$. We also refer to [17] for a study of
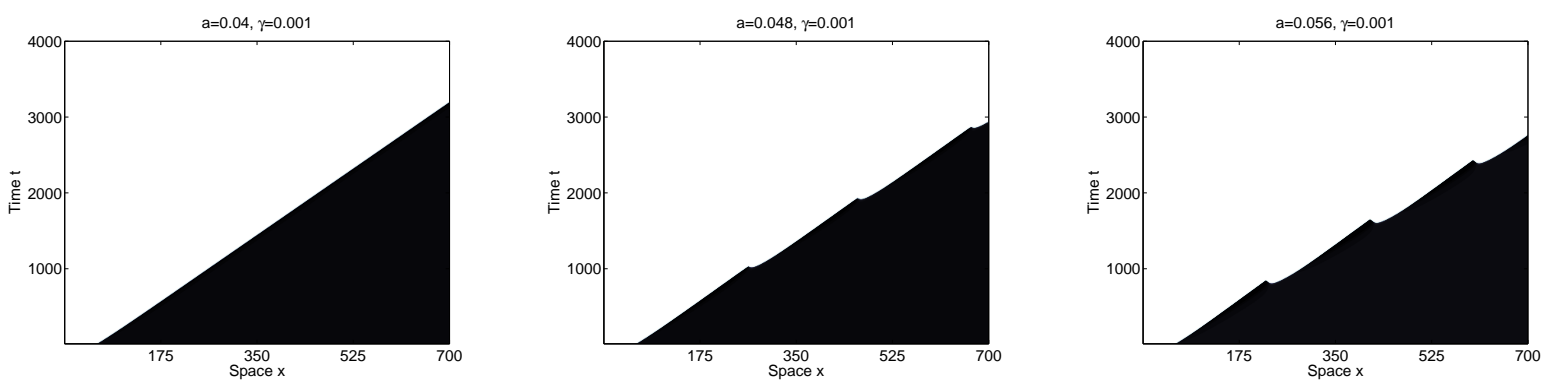

Figure 5.1: The oscillatory pushed-pulled transition for $\kappa=0.1, \gamma=0.001$ and $a=0.04,0.048,0.056$. The predicted value of the transition is $a=0.052+\mathrm{o}_{\gamma}(1)$.

propagation speeds during this crossover. We computed propagation speeds of pushed fronts by directly computing the heteroclinic connections using continuation software. On the other hand, computations as in Section 2 give predictions for linear spreading speeds. The speed measured in direct simulations agrees well with the maximum of the pushed and linear speeds computed in this fashion.

For $\kappa=0.6$, the transition only changes the speed of fronts moderately. We confirmed the predicted acceleration of the front speed for $a<a_{*}(d)$ in direct simulations. As predicted, we do not see oscillations since the two double roots at $\lambda=0$ split into two real double roots; see Section 2.4.

In both cases, we saw that the measured speed in the pulled regime underestimates the actual pulled speed, due to the predicted slow relaxation towards pulled fronts; see [10, 35] and references there.

The bistable regime - transient pattern forming fronts. Since periodic solutions in the Allen-Cahn equation are unstable, periodic patterns are unstable for small values of $\gamma$. However, this regular perturbation result is valid for bounded periods, only. For masses $a \neq 1 / 2$, one can readily show that instability holds for all periodic patterns, with arbitrary periods, since those would accumulate on a spike, a spatially homoclinic pattern, which in 
turn is unstable; see [24]. We will briefly discuss the case $a \sim 1 / 2$ in the context of Figure 5.3, below.

One is lead to predict that there are no pulled fronts with critical decay that leave behind a periodic pattern for small $\gamma$. The simulations that we show below strongly confirm this prediction. In fact, pulled fronts form a transient periodic pattern, that persists for a fixed finite time interval, before a homogeneous state is formed in the wake.

This confirms that the notion of existence of a coherent front with critical decay is a more accurate predictor than existence of a more general coherent pulled front: counting arguments (see for instance the bottom right entry in Table 4.5) do predict robust occurrence of more general coherent pulled fronts that leave behind a periodic pattern, but these fronts are not observed in direct numerical simulations.
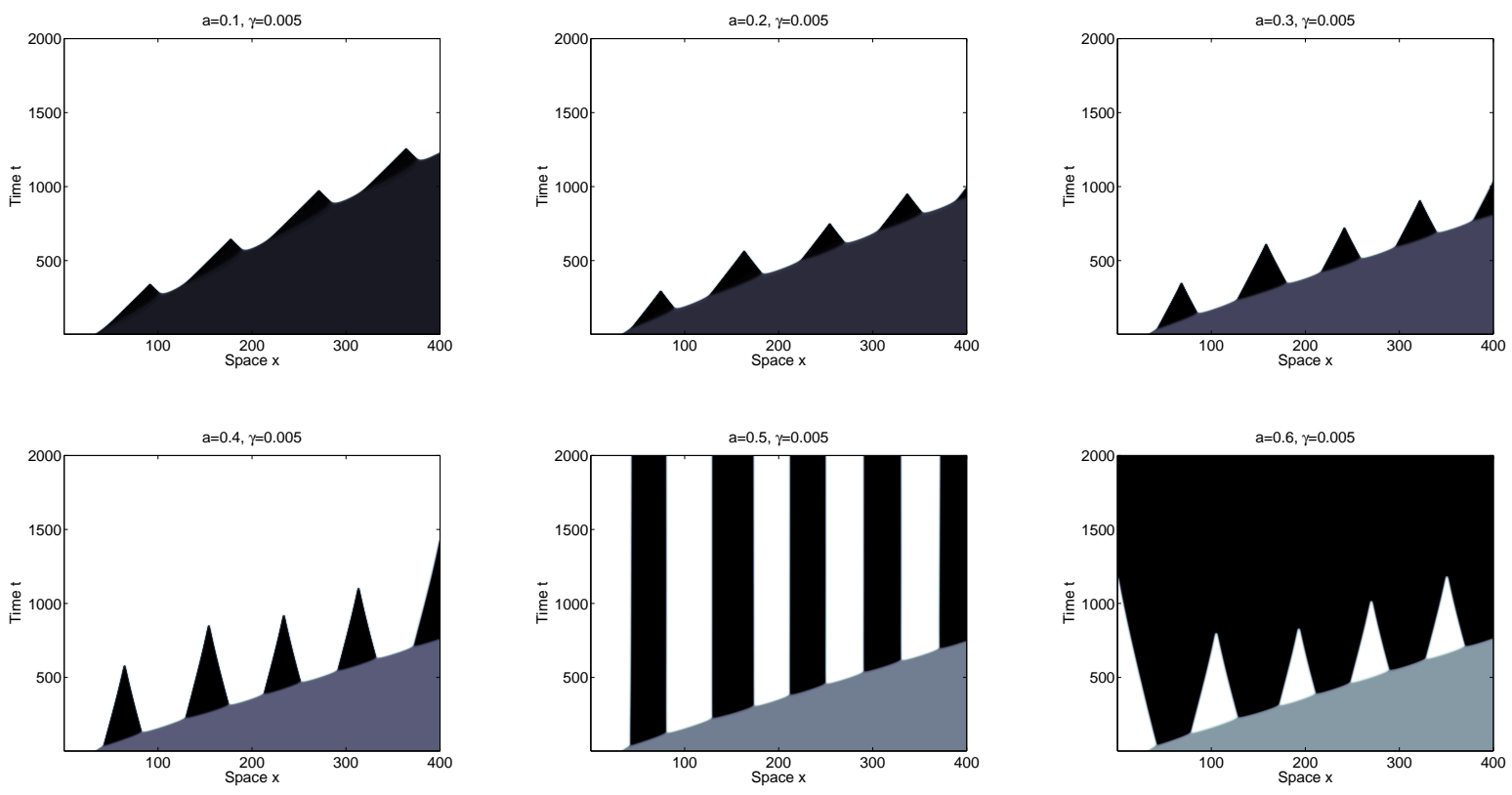

Figure 5.2: Coherent pulled fronts forming a transient pattern for $\kappa=0.1, \gamma=0.005$ and $a=$ $0.1,0.2,0.3,0.4,0.5,0.6$.

Curiously, the simulations do show a coherent pulled front leaving a pattern behind near $a=0.5$. Figure 5.3 resolves this transition in more detail. In fact, numerical and asymptotic stability analysis in this regime predict stable periodic patterns with wavelength $k_{\text {lin }}$ in a narrow wedge $a \in\left(a_{-}(\gamma), a_{+}(\gamma)\right), a_{ \pm}(0)=1 / 2$. The simulations, below, indicate that this stable pattern is in fact selected by the invasion front.

\subsection{The spinodal regime - persistent patterns}

In the parameter regime $\gamma>0.25$, spatially homogeneous equilibria are unique for fixed mass, so that unstable equilibria always coexist with at least one stable periodic pattern, $m^{\prime}(\mu)<0$. We therefore predict consistent occurrence of persistent pattern forming fronts. This is illustrated in Figure 5.4. In all cases, a wedge of periodic patterns opens behind the 

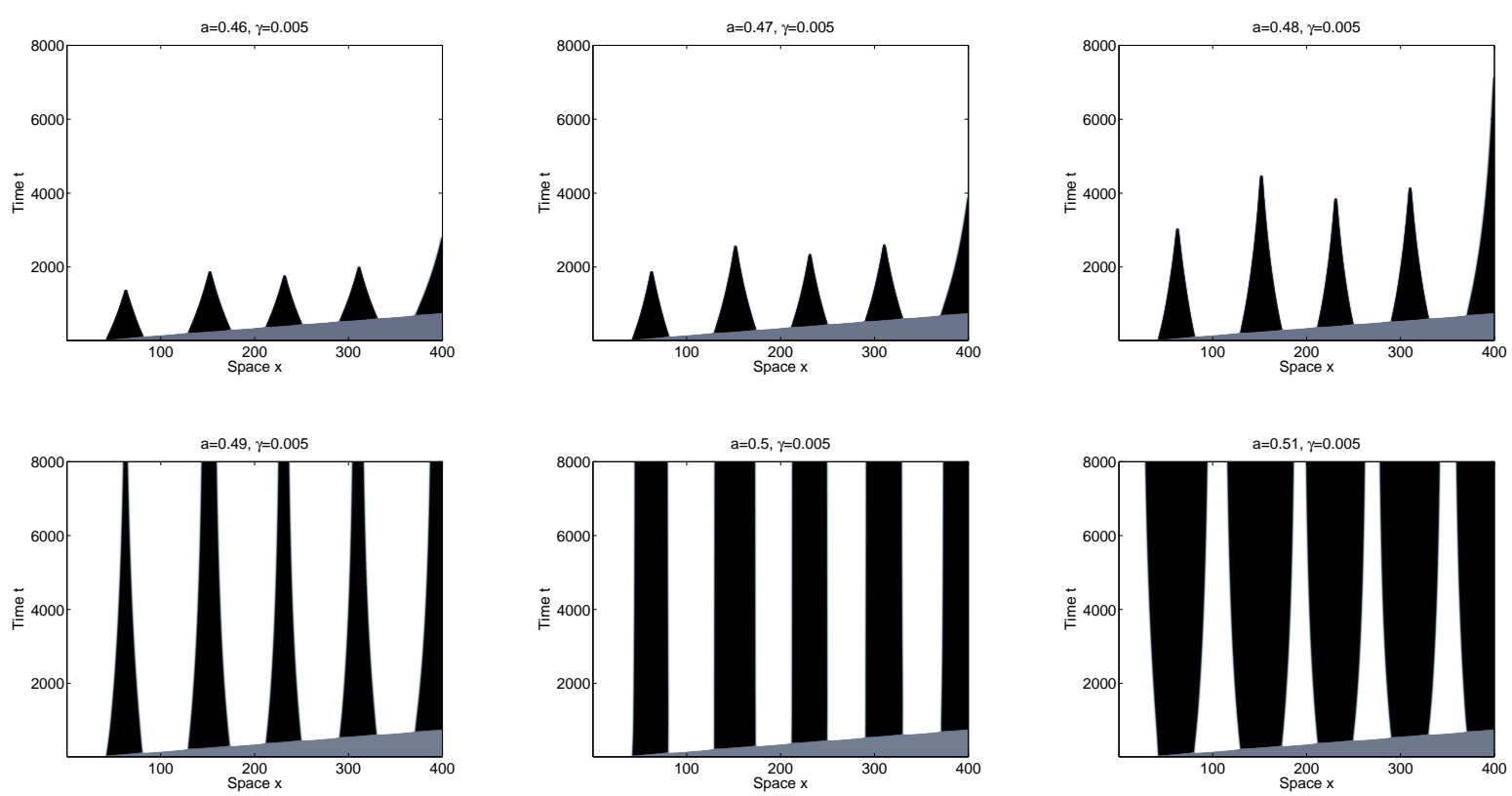

Figure 5.3: A heteroclinic codimension-one bifurcation for $\kappa=0.1, \gamma=0.005, a=0.46,0.47, \ldots, 0.51$. Near $a=0.5$ a persistent pattern forming front appears.
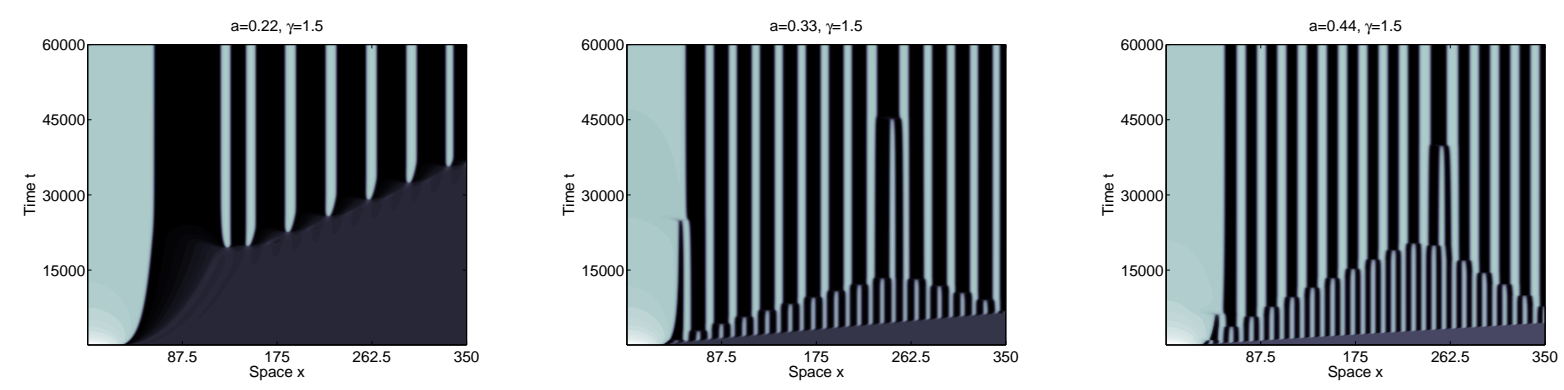

Figure 5.4: Coherent pulled fronts in the spinodal regime, $\kappa=0.1, \gamma=1.5, a=0.22,0.33,0.44$.

leading front in the space-time plot. We studied wide ranges of parameter space and have not observed transient patterns in the spinodal regime.

\subsection{From transient to persistent pattern forming fronts}

Transitions from transient to persistent patterns occur for moderately small values of $\gamma$ as $a$ increases. Figure 5.5 shows the transition for $\gamma=0.05$ near $a=0.35$. The simulations suggest that, at criticality, there exists a coherent pulled front that generates an unstable periodic pattern, consisting of a narrow band with $e \sim 0$ and a wide band with $e \sim 1$. For larger values of $a$, the pulled front creates a stable periodic pattern in its wake. For smaller values of $a$, it creates a homogeneous state. For temporal dynamics with Neumann or periodic boundary conditions, the existence of such unstable periodic patterns, whose stable manifold separates basins of attraction of stable homogeneous states and stable patterned states, is a common phenomenon in Cahn-Hilliard and phase-field equations. The stable manifold of these states 
is typically understood as the nucleation threshold. The dimension counting theory in the present work predicts that such thresholds states can be created by codimension-one pulled fronts, that is, for isolated values of the parameter $a$. Figure 5.5 shows some numerical evidence.
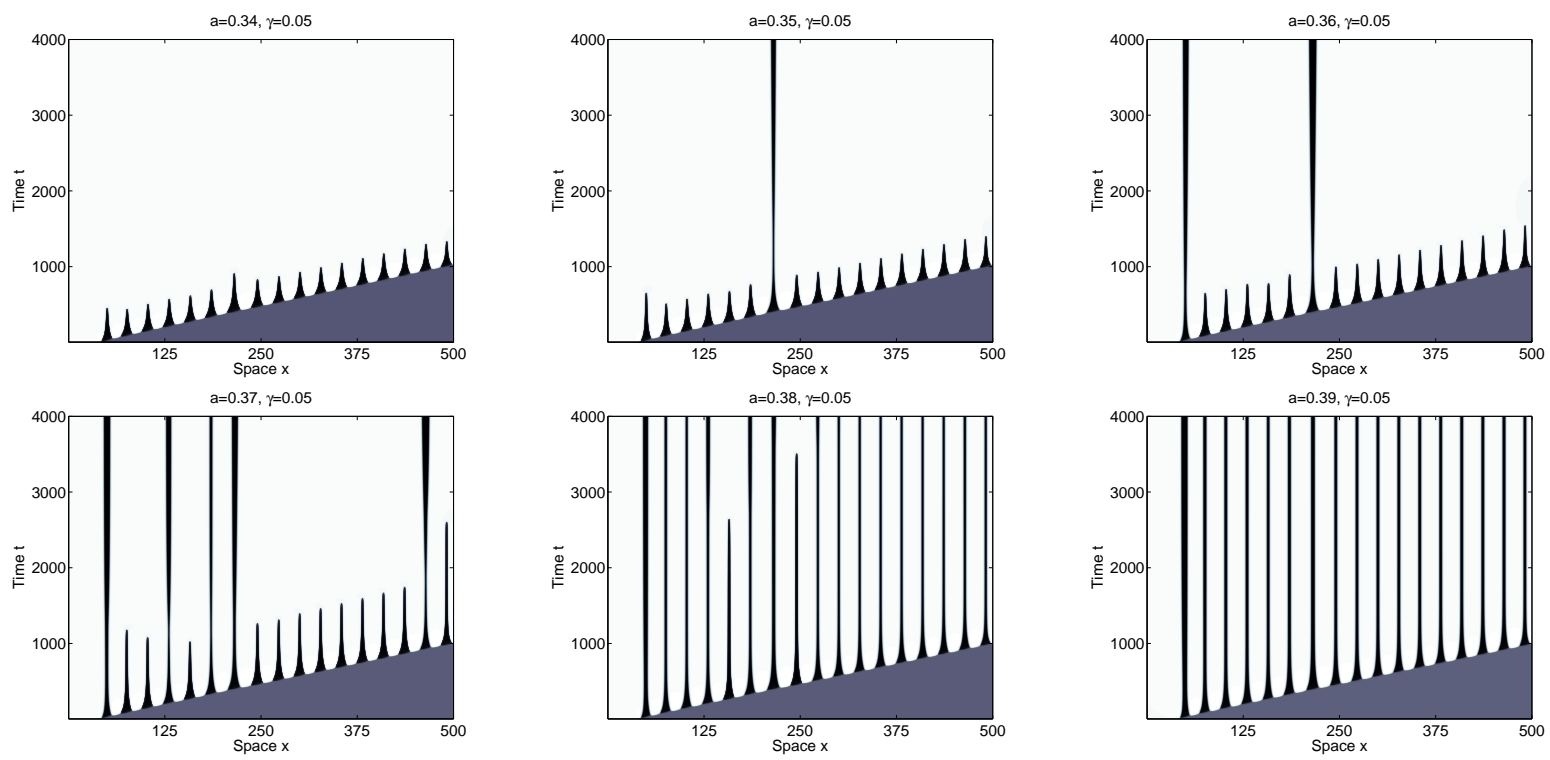

Figure 5.5: Transition from transient to persistent pattern forming fronts in the bistable regime, $\gamma=$ $0.05, \kappa=0.1$. As $a$ is increased $a=0.34,0.35,0.36,0.37,0.38,0.39$, some of the spikes that are formed in the wake begin to persist until a persistent pattern is created for larger a. We suspect that at the point of transition, a perfect persistent unstable periodic pattern is created in the wake.

\subsection{More bifurcations}

A closer inspection of patterns in the spinodal regime brings out a quite complex behavior. In the left picture of Figure 5.4, one observes a different frequency at the interface, where only some of the small spikes in the leading edge eventually form a large spike. In the other pictures, one sees coarsening in the wake of the first coherent front, typically merging neighboring spikes to double the wavelength. We explored this relation between wavenumbers in the leading edge, $k_{\text {lin }}$, and wavenumbers in the wake, $k_{\text {sel }}$, in some more detail numerically.

The spatial period-doubling transition in the leading edge is studied in more detail in Figure 5.6. One can see how a transient of patterns with half the wavelength gradually changes into a persistent pattern near $a=0.27$.

Of course, doubling of periods always suggests the presence of a cascade. Numerical simulations over large time intervals suggest that period-doubling cascades are indeed a predominant mechanism in the coarsening process that follows the primary invasion front. Figure 5.7 shows simulations on increasing time intervals, where one can observe a tertiary front that induces a second period-doubling, thus resulting in an effectively quadrupled spatial period.

Until now, we have described period-doubling mechanisms: the wavelength $2 \pi / k_{\text {sel }}$ of the pattern observed in the wake of the front is double (or quadruple) the linearly selected wavelength 

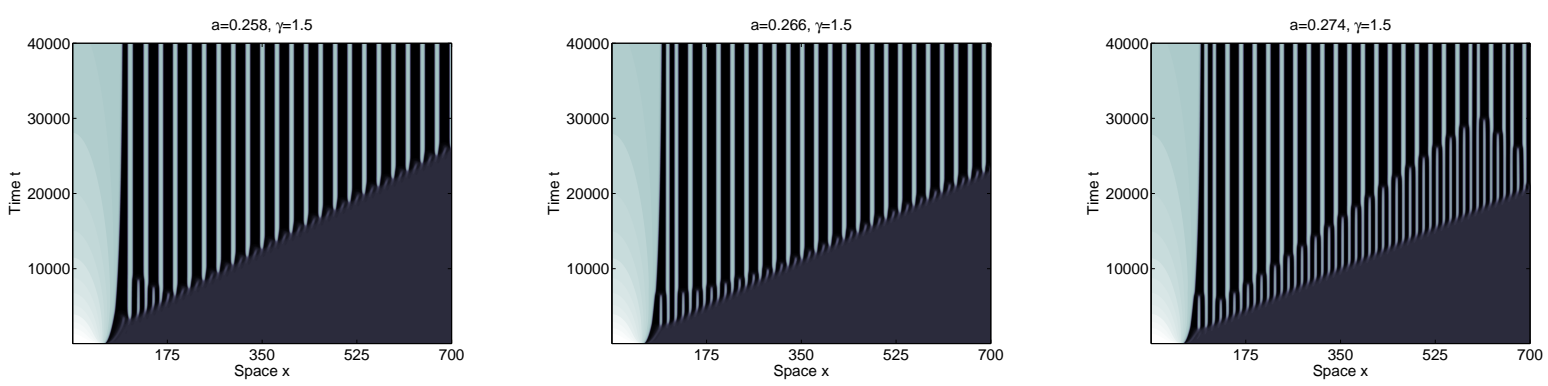

Figure 5.6: Transition from period-doubled transient to persistent patterns for coherent pulled fronts in the spinodal regime, $\kappa=0.1, \gamma=1.5, a=0.258,0.266,0.274$.
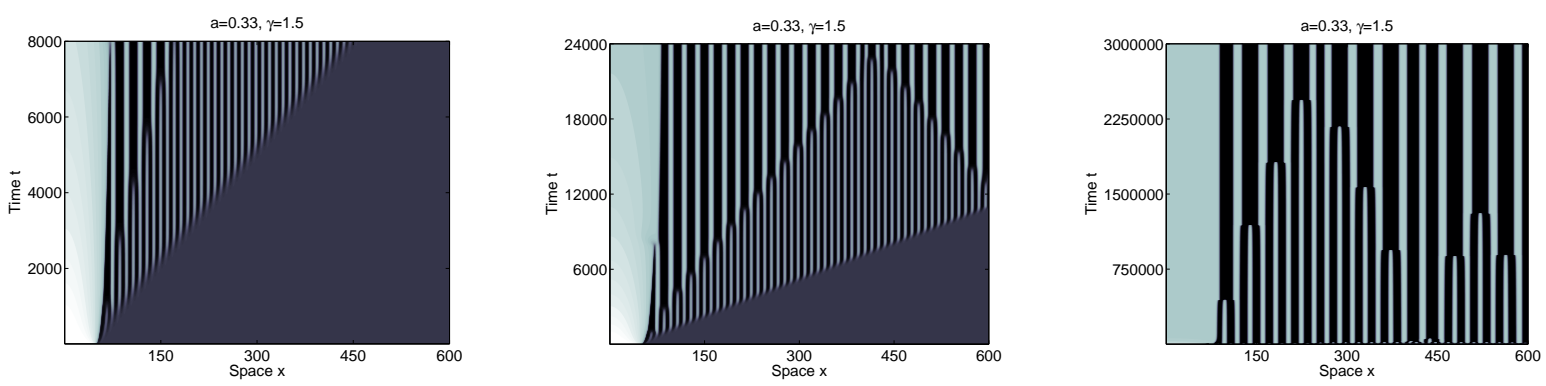

Figure 5.7: Successive spatial period-doublings leading to a quadrupled period. Parameters are chosen in the spinodal regime, $\kappa=0.1, \gamma=1.5, a=0.33$. The diagrams show space-time plots up to final times $T=8000,24000,3 \cdot 10^{6}$.

$2 \pi / k_{\text {lin }}$

$$
k_{\text {sel }}=k_{\text {lin }} / 2^{j}, \quad j=0,1,2, \ldots
$$

The doubling can occur in the leading edge, leading to transients of half-wavelength patterns in the space-time diagram, or in the wake, leading to persistent patterns in space-time diagrams. For fronts with weakly unstable equilibrium, that is, with homogeneous state close to the onset of the spinodal instability, one can observe other multiples: Figure 5.8 shows a transition

$$
k_{\text {sel }}=k_{\text {lin }} / 3 \quad \longleftrightarrow \quad k_{\text {sel }}=k_{\text {lin }} / 2
$$

in the leading edge of the pattern. We have observed other ratios, and spatial frequencylocking: the ratio $k_{\text {sel }} / k_{\text {lin }}$ changes with parameters while being constant when rational.

\subsection{Discussion}

We have presented results that can serve as guiding principles in the study of invasion problems. The system that we focused on, here, turned out to exhibit a rich phenomenology, despite its apparent simplicity and rigid variational structure.

Pushed fronts. Our first main result shows that in some parameter regimes, pushed fronts govern the invasion process. Main consequences are

- an invasion speed much faster than the linear speed; 

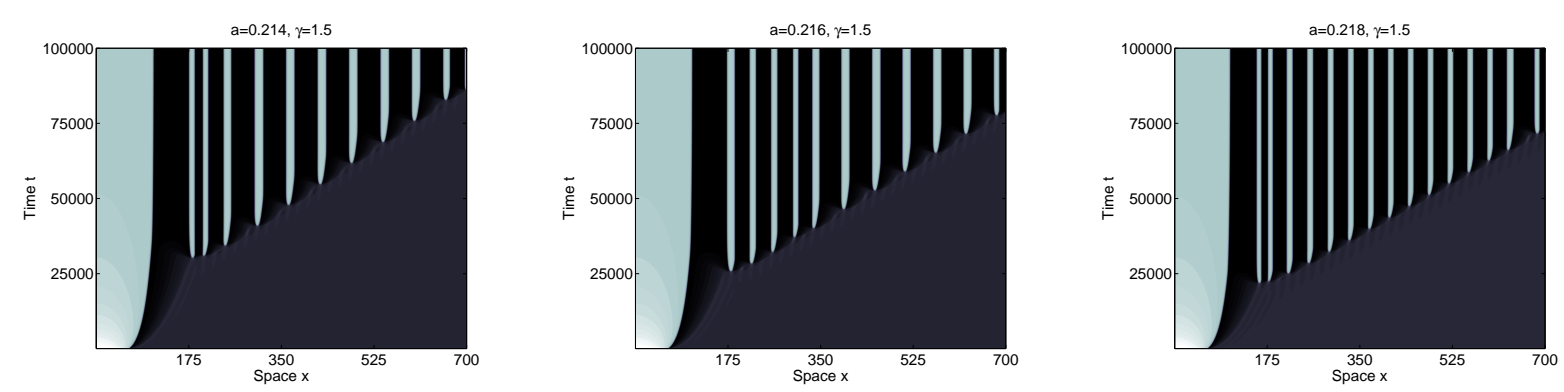

Figure 5.8: Transition from period-doubling to period-tripling of transient patterns for coherent pulled fronts in the spinodal regime, $\kappa=0.1, \gamma=1.5, a=0.214,0.216,0.218$.

- the formation of a bulk state in the wake rather than the pattern selected by the linear analysis.

We also show that the coupling of $c$ - and $e$-equation drastically increases the linear spreading for $\kappa<1 / 2$. The coupling generates oscillatory linear modes, which lead to pattern formation in the wake. As a consequence, the regime where nonlinear pushed fronts dominate pulled fronts is much smaller than in the uncoupled system $(\gamma=0)$.

Counting fronts. Our second main result can be used to qualitatively predict the existence or non-existence of invasion fronts based on dimension counting. It shows that one can expect to create an unstable pattern in the wake of an invasion front, provided that the instability of that pattern is not in resonance with the frequency of the invasion process. The creation of an unstable pattern typically leads to wedges in space-time plots, where the first invasion front is followed by a second invasion front with slower speed. We have not observed situations where the second front catches up with the primary invasion front. When the pattern in the wake possesses resonant unstable modes, there will typically not be an invasion front that creates this pattern — we observed such fronts only for isolated parameter values.

Invasion of periodic patterns. Following the counting arguments that we presented for the invasion of spatially homogeneous states, one can study the invasion of spatially periodic states. Examples are in particular the period-doubling fronts in Figure 5.7. Since periodic patterns with wavenumber $k_{*}>0$ are unstable, one can again define a linear spreading speed analogous to the spatially homogeneous case through the dispersion relation; see Section 4.5. Together with this linear spreading speed $s_{\text {lin }}$, one finds a temporal frequency $\omega_{\text {lin }}$ and a spatial wavenumber $\nu_{\text {lin }}$. It turns out that spatial period-doubling in the leading edge, $\operatorname{Im} \nu_{\text {lin }}=k_{*} / 2$, $\omega=k_{*} s_{\text {lin }} / 2$, can occur in a robust fashion. In this case, one can then follow our counting arguments and show that period-doubling invasion fronts are robust. It is an interesting question whether such spatial period-doubling is in fact the selected spreading mechanism for all (or most of) the periodic patterns in our system.

Existence of pulled fronts. Our counting arguments give criteria for robustness - once existence is known. In some cases one can expect to be able to actually prove existence exploiting the gradient structure of the system. We refer to [32], where existence and stability 
of coherent pulled fronts has been studied in the Allen-Cahn equation using Conley index theory. In fact, the traveling-wave equation (3.3) possesses a Lyapunov function,

$$
V\left(e, \theta, e_{\xi}\right)=\tilde{V}\left(e, \theta-\kappa e, e_{\xi}\right),
$$

where

$$
\tilde{V}\left(e, c, e_{\xi}\right)=\frac{\kappa}{2} e_{\xi}^{2}-G(e)+\frac{\gamma}{2(1-\kappa)}\left((c+e)^{2}-(1+\kappa) e^{2}\right)-s \frac{\gamma}{1-\kappa}(c+\kappa e),
$$

and $\mu:=(c+\kappa e)_{\xi}+s(e+c)$ is the mass function in the traveling-wave phase space. One readily calculates that

$$
\frac{\mathrm{d}}{\mathrm{d} \xi} V=-s\left(\left(e_{\xi}\right)^{2}+\frac{\gamma}{(1-\kappa) s^{2}}\left(\theta_{\xi}\right)^{2}\right) \leq 0
$$

As a consequence, all bounded solutions to (3.3) are heteroclinic orbits to equilibria.

Similarly, one can show that the modulated traveling-wave equation (4.8), which describes coherent fronts, possess a Lyapunov function,

$$
\mathcal{V}\left(e, c, e_{\xi}, c_{\xi}\right)=\int_{0}^{2 \pi}\left(\frac{\kappa}{2} e_{\xi}^{2}-G(e)+\frac{\kappa \omega}{s} e_{\xi} e_{\tau}-\frac{\gamma}{s(1-\kappa)}\left[(c+\kappa e)(c+\kappa e)_{\xi}+\kappa e c\right]\right) \mathrm{d} \tau,
$$

so that along solutions,

$$
\frac{\mathrm{d}}{\mathrm{d} \xi} \mathcal{V}=-\frac{1}{s}\left(s e_{\xi}+\omega e_{\tau}\right)^{2}-\frac{\gamma}{s(1-\kappa)} \theta_{\xi}^{2} \leq 0
$$

In particular, all bounded solutions converge to stationary patterns, $s e_{\xi}+\omega e_{\tau}=e_{t}=0$, $\theta_{\xi}=0$.

Although the traveling-wave equation to phase-field systems has been studied quite extensively in the literature, it seems that the existence of a Lyapunov function, even for pure traveling waves, (3.3), has not been noted previously.

Exploiting the Lyapunov function in order to prove existence of coherent pulled fronts will be subject of future work.

Bifurcations. We have presented both numerical and theoretical evidence for the presence of a number of bifurcations that change the pattern in the wake in a qualitative fashion. It would be interesting to study some of those bifurcations in more detail theoretically. For instance, it would be interesting to analyze the pushed-pulled transition theoretically, thus deriving expansions in $\gamma$ for the transition point $a_{*}(\kappa)$ and possibly prove existence of transient pattern forming fronts. In a related direction, we plan to investigate the period-doubling bifurcations both in the leading edge and in the wake.

Summary. Our analysis gives a first rough outline of the complexity that arises from spatially localized perturbations of unstable states in extended systems. Although the systems that we study possess a gradient structure, the spatial complexity involves complicated dynamic bifurcations, such as Hopf bifurcations, period-doublings, and frequency locking. There are numerous open questions, but we hope that a complete description of pattern selection through front invasion will be possible in the near future. 


\section{References}

[1] P. Bates, P. Fife, R. Gardner, and C.K.R.T. Jones. The existence of travelling wave solutions of a generalized phase-field model. SIAM J. Math. Anal. 28 (1997), 60-93.

[2] J. Bellay and A. Scheel. Coherent structures near the boundary between excitable and oscillatory media. Dyn. Syst., to appear.

[3] L. Brevdo and T.J. Bridges. Absolute and convective instabilities of spatially periodic flows. Philos. Trans. Roy. Soc. London Ser. A 354 (1996), 1027-1064.

[4] R.J. Briggs. Electron-stream interaction with plasmas. MIT Press, Cambridge, 1964.

[5] G. Caginalp. The dynamics of a conserved phase field system: Stefan-like, Hele-Shaw, and CahnHilliard models as asymptotic limits. IMA J. Appl. Math. 44 (1990), 77-94.

[6] G. Caginalp and Y. Nishiura, The existence of travelling waves for phase field equations and convergence to sharp interface models in the singular limit. Quart. Appl. Math. 49 (1991), 147162.

[7] M. Droz. Recent Theoretical Developments on the Formation of Liesegang Patterns. J. Stat. Physics 101 (2000), 509-519.

[8] C. Dupaix, D. Hilhorst, I. Kostin. The viscous Cahn-Hilliard equation as a limit of the phase field model: lower semicontinuity of the attractor. J. Dynam. Differential Equations 11 (1999), $333-353$.

[9] U. Ebert and W. van Saarloos. Breakdown of the standard Perturbation Theory and Moving Boundary Approximation for "Pulled" Fronts. Phys. Rep. 337 (2000), 139-156.

[10] U. Ebert and W. van Saarloos. Front propagation into unstable states: universal algebraic convergence towards uniformly translating pulled fronts. Physica D 146 (2000), 1-99.

[11] J. Eilbeck, J. Furter, and M. Grinfeld. On a stationary state characterization of transition from spinodal decomposition to nucleation behaviour in the Cahn-Hilliard model of phase separation. Phys. Lett. A 135 (1989), 272-275.

[12] B. Fiedler and A. Scheel. Spatio-Temporal Dynamics of Reaction-Diffusion Patterns. In Trends in Nonlinear Analysis, M. Kirkilionis, S. Krmker, R. Rannacher, F. Tomi (Eds.), Springer-Verlag, Berlin, 2003.

[13] P. Fife. Pattern formation in gradient systems. Handbook of dynamical systems, Vol. 2, 677-722, North-Holland, Amsterdam, 2002.

[14] K. Glasner Traveling waves in rapid solidification. Electron. J. Differential Equations 16 (2000), $1-28$.

[15] K. Glasner. Rapid growth and critical behaviour in phase field models of solidification. European J. Appl. Math. 12 (2001), no. 1, 39-56.

[16] K. Glasner and R. Almgren. Dual fronts in a phase field model. Phys. D 146 (2000), 328-340.

[17] R. Goh, S. Mesuro, and A. Scheel. Coherent structures in reaction-diffusion models for precipitation. To appear in Precipitation patterns in reaction-diffusion systems, Res. SIGNPOST. 
[18] M. Grinfeld, J. Furter, and J. Eilbeck. A monotonicity theorem and its application to stationary solutions of the phase field model. IMA J. Appl. Math. 49 (1992), 61-72.

[19] D. Hilhorst, R. van der Hout, M. Mimura, and I. Ohnishi. A Mathematical Study of the OneDimensional Keller and Rubinow Model for Liesegang Bands. J. Stat. Phys. 135 (2009), 107-132.

[20] G. Iooss and A. Mielke. Bifurcating time-periodic solutions of Navier-Stokes equations in infinite cylinders. J. Nonlinear Sci. 1 (1991), 107-146.

[21] J.B. Keller and S. Rubinow. Recurrent precipitation and Liesegang rings. J. Chem. Phys. 74 (1981), 5000-5007.

[22] K. Kirchgässner. Wave-solutions of reversible systems and applications. J. Differential Equations 45 (1982), 113-127.

[23] D. Peterhof, B. Sandstede, and A. Scheel. Exponential dichotomies for solitary wave solutions of semilinear elliptic equations on infinite cylinders. J. Diff. Eqns. 140 (1997), 266-308.

[24] A. Pogan and A. Scheel. Instability of Spikes in the Presence of Conservation Laws. Z. Angew. Math. Phys., to appear.

[25] A. Pogan and A. Scheel. Stability and instability of patterns in the presence of conservation laws, in preparation.

[26] J.D.M. Rademacher, B. Sandstede, and A. Scheel. Computing absolute and essential spectra using continuation. Physica D 229 (2007), 166-183.

[27] B. Sandstede and A. Scheel. Absolute and convective instabilities of waves on unbounded and large bounded domains. Physica D 145 (2000), 233-277.

[28] B. Sandstede and A. Scheel. On the structure of spectra of modulated travelling waves. Math. Nachr. 232 (2001), 39-93.

[29] B. Sandstede and A. Scheel. Defects in oscillatory media: toward a classification. SIAM J. Appl. Dyn. Syst. 3 (2004), 1-68.

[30] B. Sandstede and A. Scheel. Relative Morse indices, Fredholm indices, and group velocities. Discrete Contin. Dyn. Syst. 20 (2008), 139-158.

[31] A. Scheel. Radially symmetric patterns of reaction-diffusion systems. Mem. Amer. Math. Soc. 165 (2003).

[32] A. Scheel. Coarsening fronts. Arch. Ration. Mech. Anal. 181 (2006), 505-534.

[33] J. Sherratt, M. Smith, and J. Rademacher. Locating the transition from periodic oscillations to spatiotemporal chaos in the wake of invasion. Proc. Natl. Acad. Sci. 106 (2009), 10890-10895.

[34] M. Smith, J. Rademacher, and J. Sherratt. Absolute stability of wavetrains can explain spatiotemporal dynamics in reaction-diffusion systems of lambda-omega type. SIAM J. Appl. Dyn. Syst. 8 (2009), 1136-1159.

[35] W. Van Saarloos. Front propagation into unstable states Phys. Rep. 386 (2003), 29-222.

[36] M. Zukerman, R. Kupferman, O. Shochet, and E. Ben-Jacob. Concentric decomposition during rapid compact growth. Physica D 90 (1996), 295-305. 